ARTICLE

https://doi.org/10.1038/s41467-019-11386-4

\title{
Phc2 controls hematopoietic stem and progenitor cell mobilization from bone marrow by repressing Vcam1 expression
}

Joonbeom Bae ${ }^{1,8}$, Sang-Pil Choi ${ }^{1,8}$, Kyoichi Isono ${ }^{2}$, Ji Yoon Lee ${ }^{3}$, Si-Won Park ${ }^{1}$, Chang-Yong Choi ${ }^{1}$, Jihye Han ${ }^{1}$, Sang-Hoon Kim¹, Han-Hyoung Lee ${ }^{1}$, Kyungmin Park1, Hyun Yong Jin ${ }^{4}$, Suk Jun Lee ${ }^{5}$, Chung-Gyu Park (1) ${ }^{6}$, Haruhiko Koseki ${ }^{7}$, Young Sik Lee (i) ${ }^{1} \&$ Taehoon Chun (i) ${ }^{1}$

The timely mobilization of hematopoietic stem and progenitor cells (HSPCs) is essential for maintaining hematopoietic and tissue leukocyte homeostasis. Understanding how HSPCs migrate between bone marrow (BM) and peripheral tissues is of great significance in the clinical setting, where therapeutic strategies for modulating their migration capacity determine the clinical outcome. Here, we identify an epigenetic regulator, Phc2, as a critical modulator of HSPC trafficking. The genetic ablation of Phc2 in mice causes a severe defect in HSPC mobilization through the derepression of Vcam1 in bone marrow stromal cells (BMSCs), ultimately leading to a systemic immunodeficiency. Moreover, the pharmacological inhibition of VCAM-1 in Phc2-deficient mice reverses the symptoms. We further determine that Phc2-dependent Vcam1 repression in BMSCs is mediated by the epigenetic regulation of H3K27me3 and H2AK119ub. Together, our data demonstrate a cell-extrinsic role for Phc2 in controlling the mobilization of HSPCs by finely tuning their bone marrow niche.

\footnotetext{
${ }^{1}$ Department of Biotechnology, College of Life Sciences and Biotechnology, Korea University, Seoul 02841, Republic of Korea. ${ }^{2}$ Laboratory Animal Center, Wakayama Medical University, Wakayama 641-8509, Japan. ${ }^{3}$ Department of Biomedical Science, CHA University, Seongnam, Gyounggi-do 13488, Republic of Korea. ${ }^{4}$ Department of Urology, School of Medicine, University of California, San Francisco, San Francisco, CA 94158, USA. ${ }^{5}$ Department of Biomedical Laboratory Science, College of Health Science, Cheongju University, Cheongju-si 28503, Republic of Korea. ${ }^{6}$ Department of Microbiology and Immunology, Cancer Research Institute and Xenotransplantation Research Center, Seoul National University College of Medicine, Seoul 03087, Republic of Korea.

${ }^{7}$ Laboratory for Developmental Genetics, RIKEN Center for Integrative Medical Sciences, Yokohama 230-0045, Japan. ${ }^{8}$ These authors contributed equally: Joonbeom Bae, Sang-Pil Choi. Correspondence and requests for materials should be addressed to T.C. (email: tchun@korea.ac.kr)
} 
ematopoietic stem and progenitor cells (HSPCs) are rare populations of cells that can sustain the hematopoietic system by continuously replenishing blood cells ${ }^{1,2}$. Postnatally, most HSPCs engraft and reside in the BM niche, but a small fraction of these cells is mobilized and migrate into the peripheral blood $(\mathrm{PB})$ to reconstitute the hematopoietic system under specific signaling cues ${ }^{1,3,4}$. Therefore, identifying key regulators that critically control HSPC mobilization and understanding their underlying mechanisms have been of significant interest to facilitate BM transplantation efficiency and to therapeutically inhibit the malignant migration of the transformed hematopoietic cells into peripheral tissues ${ }^{5}$. Indeed, several BM niche proteins, including stem cell factor (kit ligand), CXCL12 (SDF-1 $\alpha$ ), and $\beta 1$ integrin have been identified as key signaling components to control the maturation of HSPCs, their migration into the circulation and their homing to the BM by mediating the interaction between HSPCs and bone marrow stromal cells (BMSCs) ${ }^{2,5-7}$. However, the molecular pathways that regulate the expression of these $\mathrm{BM}$ niche proteins remain unclear.

Polycomb group (PcG) proteins function as transcriptional repressors of target genes by mainly modulating histone methylation ${ }^{8}$. These PcG proteins can be divided into two functionally distinct complexes: polycomb repressor complex 1 (PRC1) and polycomb repressor complex 2 (PRC2). PRC2 induces the formation of trimethylated histone $\mathrm{H} 3$ at lysine 27 (H3K27me3) through methyltransferase activity of Ezh proteins which are component proteins in PRC2 complex 9 . The canonical PRC1 then recognizes and binds to $\mathrm{H} 3 \mathrm{~K} 27 \mathrm{me} 3$ to sustain the transcriptional repression of a target gene $8,10,11$. Both canonical and noncanonical PRC1 complexes can cause additional transcriptional silencing as E3 ubiquitin ligases by forming monoubiquitylated histone $\mathrm{H} 2 \mathrm{~A}$ at lysine 119 (H2AK119ub) ${ }^{8,12,13}$.

In this study, we demonstrate that Phc2, a component of the canonical PRC1, regulates HSPC mobilization through the repression of Vcam 1 expression by enhancing both H3K27me3 and H2AK119ub in BMSCs. Therefore, Phc2 deficiency causes a severe HSPC mobilization defect via the derepression of Vcam1 in BMSCs, and the pharmacological inhibition of VCAM-1 in BMSCs significantly reverses the symptoms of Phc2-deficient mice. These data demonstrate the critical cell-extrinsic role of Phc2 in controlling HSPC mobilization and provide the first evidence of epigenetic control over HSPC mobilization.

\section{Results}

Phc2 deficiency leads to a defect in HSPC mobilization. As an initial step to elucidate the functional role of Phc2 during hematopoiesis, we characterized immune phenotypes of $\mathrm{Phc2} \mathrm{C}^{-1}-$ (KO) mice $^{14}$ compared to those of $P h c 2^{+/+}$(wild-type, WT) mice. We observed macroscopic abnormalities in the thymus and peripheral lymphoid organs of KO mice compared to those of WT mice. Both the thymus and spleen of the KO mice maintained normal architecture, but these organs were smaller than those of the WT mice (Fig. 1a, b). Consistent with this observation, the absolute numbers of immune cells and their precursors in the $\mathrm{PB}$, thymus, spleen, and liver of the $\mathrm{KO}$ mice were significantly reduced compared to those of the WT mice (Tables 1 and 2, Fig. 1c). Notably, the absolute number of early T-cell precursors (ETPs) in the thymus or immature $\mathrm{B}$ cells in the spleen was significantly lower in the KO mice relative to the WT mice (Table 1).

Unexpectedly, the absolute numbers and relative ratios of HSPCs, early progenitor cells, and immune cells in the BM were similar among all examined mice (Table 1 and Fig. 1c, d). Additionally, the loss of Phc2 did not influence the cell cycle progression or apoptosis of HSPCs (Fig. 1e, f), and both the WT and KO BM-resident HSPCs exhibited no difference in terms of their ability to generate multipotential or myeloid progenitor cells (Fig. 1g). The absolute numbers of WT and KO B cells in the BM at each developmental stage were not significantly different either (Fig. 1h). Given that no functional defect was evident in the HSPCs of the BM but that there was a shortage of ETPs and immature B cells from the BM, we postulated that a Phc2 deficiency could lead to a systemic immunodeficiency due to a defect in HSPC migration into the circulation.

To test this hypothesis, we examined the numbers of circulating HSPCs from WT and KO mice using a colonyforming unit (CFU) assay. The absolute numbers of clonogenic progenitors in the $\mathrm{PB}$ and spleen were decreased by approximately half in the $\mathrm{KO}$ mice compared to the WT mice, whereas the absolute numbers of clonogenic progenitors in the $\mathrm{BM}$ were comparable between the WT and KO mice (Supplementary Fig. 1). To confirm this result, we performed in vivo HSPC mobilization assays using two commonly used mobilization regimens for therapy, granulocyte colony-stimulating factor (GCSF) and AMD3100 (CXCR4 antagonist) ${ }^{15-18}$. Five days after GCSF treatment, the absolute numbers of white blood cells (WBCs) and splenocytes from the $\mathrm{KO}$ mice remained significantly lower than those from the WT mice (Fig. 2a, b). The spleen size of the $\mathrm{KO}$ mice also remained much smaller than that of the WT mice after G-CSF treatment (Fig. 2b). Consistent with these results, both the frequencies and absolute numbers of $\mathrm{Lin}^{-} \mathrm{Sca}-1^{+} \mathrm{c}-\mathrm{kit}^{+}$ cells (LSK cells) in the PB and spleen were significantly lower in the KO mice than in the WT mice (Supplementary Fig. 2). Likewise, the absolute numbers of clonogenic progenitors in the $\mathrm{PB}$ and spleen of the $\mathrm{KO}$ mice were significantly reduced compared to those of the WT mice (Fig. 2c). However, the frequencies and absolute numbers of LSK cells in the BM were comparable between the WT and KO mice (Supplementary Fig. 2). Moreover, the absolute numbers of clonogenic progenitors in the BM were not significantly different between the WT and KO mice (Fig. 2c). When we used an AMD3100 as a mobilizing agent of LSK, we still observed that the migration of KO LSK into the periphery is statistically decreased compared with that of WT LSK (Fig. 2d, e). However, the defective migration of KO LSK after treatment of AMD3100 is relatively modest when we compared with results from G-CSF treatment (Fig. 2d, e).

To remove circulating HSPCs and visualize neo-HSPC mobilization from BM, we further treated WT and $\mathrm{KO}$ mice with 5-fluorouracil (5-FU), a chemotherapeutic agent that depletes circulating HSPCs and promotes HSPC release from BM during hematopoietic repopulation ${ }^{19,20}$. The absolute numbers of WBCs and splenocytes recovered from $\mathrm{KO}$ mice were lower than those recovered from WT mice starting on day 8 after 5-FU treatment (Fig. 2f, g). The most prominent differences in the absolute numbers of WBCs and splenocytes or the size of the spleen between WT and KO mice was observed on day 16 after 5-FU treatment (Fig. 2f, g). On day 16 after 5-FU treatment, the frequency and the absolute number of recovered LSK cells and the absolute number of recovered clonogenic progenitors in the $\mathrm{PB}$ and spleen were significantly lower in $\mathrm{KO}$ mice than in WT mice, whereas those in the BM were comparable between the WT and KO mice (Fig. 2h, Supplementary Fig. 3). Taken together, these results indicate that $\mathrm{Phc} 2$ deficiency causes a defect in HSPC mobilization from the BM into the periphery.

Phc2-/- BMSCs cause a defect in HSPC mobilization. To identify which cell type in the BM niche contributed to the defect in HSPC release into the circulation of $\mathrm{KO}$ mice, we adoptively transferred LSK cells isolated from WT CD45.1 mice into 
a

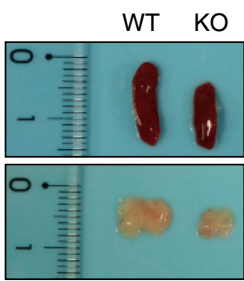

b

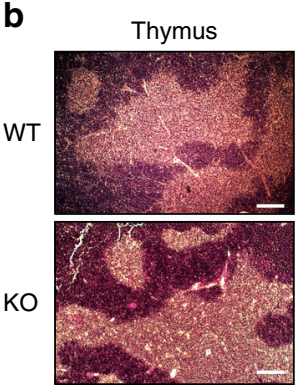

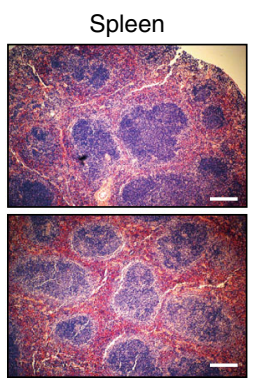

C

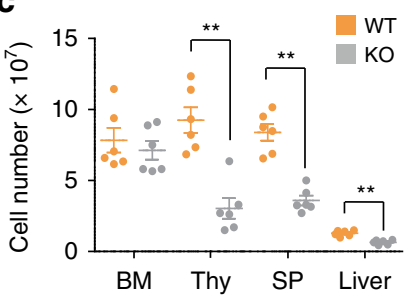

e

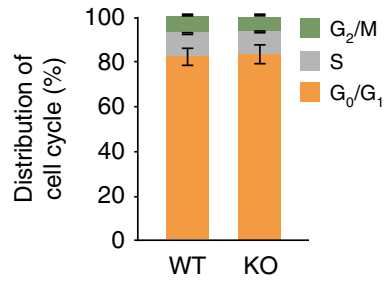

h

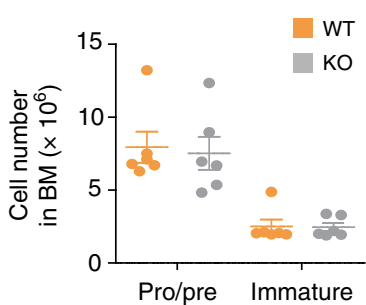

Fig. 1 Phc2 deficiency leads to immune cell deficiency without intrinsic defects in HSPCs. a Representative images of spleens and thymi from WT and KO mice. $n=6$. $\mathbf{b}$ Normal architecture of the thymus and spleen of the $\mathrm{KO}$ mouse. Representative hematoxylin and eosin staining images of spleens and thymi from WT and KO mice. $n=6$. Scale bars: $200 \mu \mathrm{m}$. c Absolute number of immune cells in the BM, thymus (Thy), spleen (SP), and liver of WT or KO mice. $n$

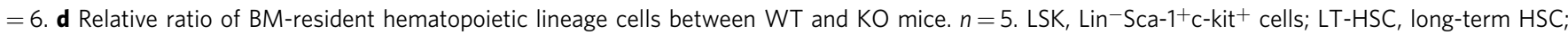
SLAM-HSC, Lin-CD41-CD48-CD150+ cells; ST-HSC, short-term HSC; MPP, multipotent progenitors; CMP, common myeloid progenitors; GMP granulocyte/monocyte progenitors; MEP, megakaryocyte/erythrocyte progenitors; CLP, common lymphoid progenitors; Myeloid, myeloid cells. e Cell cycle status of BM-resident LSK cells from WT and KO BM. $n=4$. f Percentage of apoptosis for BM-resident LSK cells from WT and KO mice. $n=3$. $\mathbf{g}$ BMresident clonogenic progenitors from WT and KO mice were assessed by CFU assays. $n=5$. CFU-GM, CFU-granulocyte and/or macrophage; BFU-E, erythroid burst-forming units, CFU-GEMM, CFU-granulocyte/erythrocyte/monocyte/megakaryocyte. $\mathbf{h}$ Absolute number of BM-resident B cell subpopulations from WT and KO mice $(n=6)$. Pro/pre, pro-B cells/pre-B cells; Immature, immature B cells. Statistical significance was assessed by twotailed Student's $t$-test. ${ }^{\star \star} P<0.01$. All data are presented as the mean \pm SEM. Source data are provided as a Source Data File

irradiated WT or KO CD45.2 recipient mice (Fig. 3a). KO recipients showed impaired repopulation of donor-derived immune cells in the thymus and spleen (Fig. 3b, c). However, the absolute numbers of LSK cells and lineage-committed progenitors ( $\mathrm{Lin}^{-}{ }^{-}$kit $^{+}$cells; LK cells) in the BM of $\mathrm{KO}$ recipients were comparable to those in the WT recipients (Fig. $3 \mathrm{~b}-\mathrm{d}$ ). To confirm these observations, irradiated WT CD45.1 mice were reconstituted with LSK cells isolated from WT or KO CD45.2 mice (Fig. 3e). The origin of the donor LSK cells did not affect the repopulation of leukocytes in WT recipients (Fig. 3f, g). The origin of the donor LSK cells did not affect the absolute number of LSK cells or LK cells in the BM of WT recipients either (Fig. 3h). We also performed a serial competitive BM repopulation assay to further determine whether KO HSPCs had an intrinsic defect. We found that KO HSPCs had the same ability to reconstitute immune cells as WT HSPCs (Supplementary Fig. 4). This strongly suggests that the defect in HSPC release into the circulation in $\mathrm{KO}$ mice is caused by an extrinsic factor from BMSCs within BM niches.

To confirm this result, we performed an in vivo homing assay with CFSE-labeled WT and KO LSK cells. The number of CFSE ${ }^{+}$
LSK cells homing to the BM was higher among the $\mathrm{KO}$ recipients than among the WT recipients (Fig. 4a). Such an increase in the short-term migration of $\mathrm{CFSE}^{+}$LSK cells into the $\mathrm{BM}$ of $\mathrm{KO}$ recipients led to fewer $\mathrm{CFSE}^{+}$LSK cells homing to the PB and spleen of the KO recipients (Fig. 4a). Accordingly, the frequency of clonogenic progenitors in the $\mathrm{BM}$ of the $\mathrm{KO}$ recipients was increased, whereas the frequencies of clonogenic progenitors in the $\mathrm{PB}$ and spleen of the KO recipients were decreased compared to those of the WT recipients (Fig. 4b, Supplementary Fig. 5). We next measured the capacity of WT and KO LSK cells to transmigrate across their BMSCs using a trans-stromal migration assay. The number of LSK cells transmigrating across KO BMSCs was smaller than the number of LSK cells transmigrating across WT BMSCs (Fig. 4c, Supplementary Fig. 6). In contrast, the number of LSK cells adhering to KO BMSCs was greater than the number of LSK cells adhering to WT BMSCs (Fig. 4d, Supplementary Fig. 6). Collectively, these data demonstrate that an extrinsic factor from BMSCs causes HSPCs to adhere more strongly to BMSCs, ultimately leading to the defective mobilization of HSPCs from the BM into the periphery in $\mathrm{KO}$ mice. 


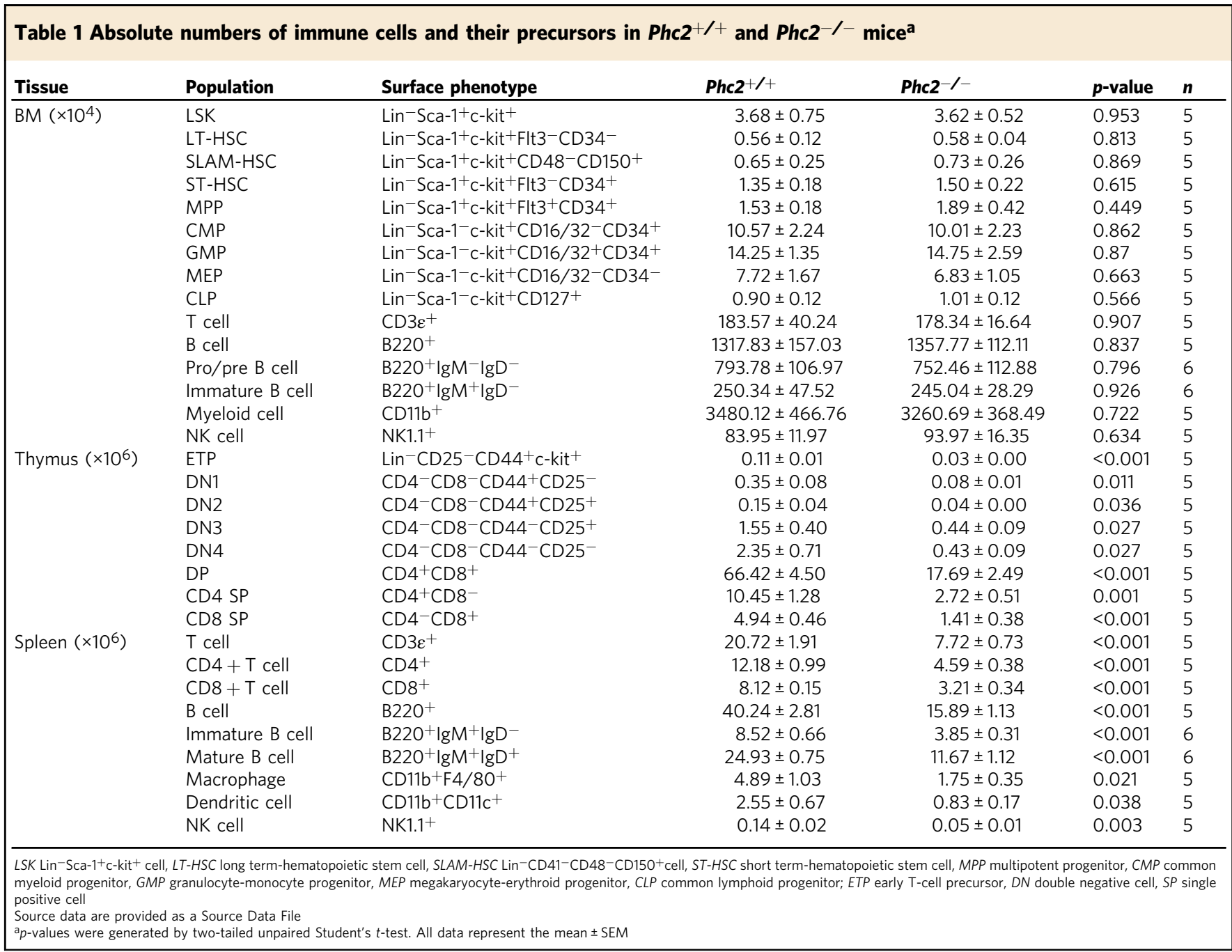

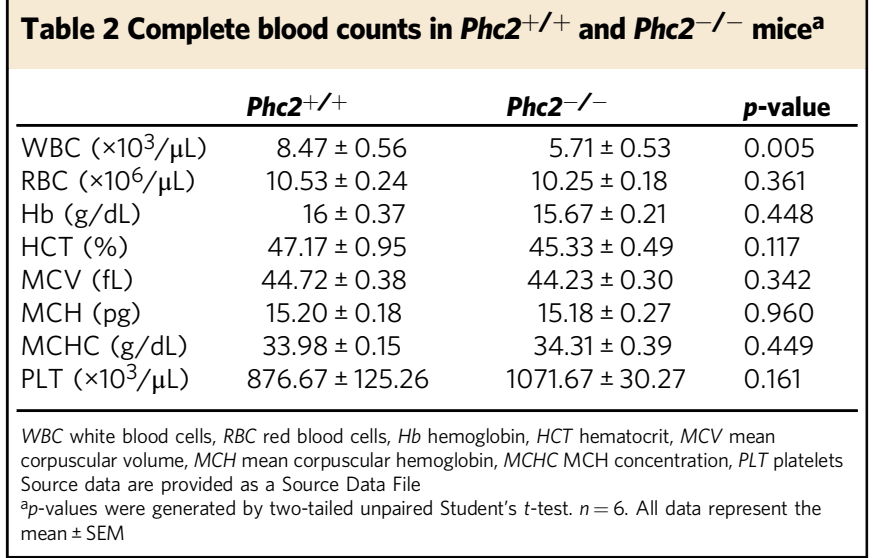

PcG proteins repress Vcam1 gene expression in BMSCs. To understand the molecular mechanisms through which PcG strengthens the adhesion properties of HSPCs to BMSCs, we first investigated the compositions and architectures of BM niches in WT and KO mice and measured the expression levels of candidate genes that are known to be involved in these processes. Endothelial cells, mesenchymal stem cells, and osteoblasts are major BMSCs found in BM niches ${ }^{21-23}$. Importantly, neither a change in BMSC composition nor an abnormal architecture was found in the KO BM niches (Supplementary Figs. 7 and 8). Next, we examined the expression patterns of soluble factors and cell surface molecules that might regulate HSPC mobilization in WT and $\mathrm{KO} \mathrm{BMSCs}{ }^{2,7}$ (Fig. 5a, Supplementary Fig. 9). Intriguingly, both the mRNA and protein levels of VCAM-1 were significantly increased in KO BMSCs compared to WT BMSCs (Fig. 5a, b). Flow cytometry analysis further confirmed that the expression of VCAM-1 was significantly higher in KO BMSCs than in WT BMSCs (Fig. 5c). Consistent with this observation, Vcam1 mRNA levels were significantly elevated in activated $\mathrm{KO}$ peritoneal macrophages compared to those of WT (Supplementary Fig. 10).

Taken together, these results indicate that Phc2 may repress Vcam1 expression in BMSCs. To further examine Phc2dependent Vcam1 repression, we knocked down Phc2 expression in BMSCs (OP9 cells) and concurrently monitored Vcam1 expression levels under treatment with TNF- $\alpha$, a well-known inducer of Vcam 1 transcription ${ }^{24}$ (Fig. 5d). As expected, TNF- $\alpha$ treatment induced the expression of Vcam 1 at $3 \mathrm{~h}$ post-treatment. However, this was significantly exacerbated when Phc2 was depleted, and thus, the VCAM-1 level was not dampened up to $12 \mathrm{~h}$ post-TNF- $\alpha$ treatment (Fig. 5e, f). Overall, these results suggest that Phc2 represses the expression of Vcam1 in BMSCs at the transcriptional level.

To demonstrate a role for Phc2 in the transcriptional repression of Vcam1, we performed a chromatin immunoprecipitation (ChIP) assay with seven primer pairs covering the 
a
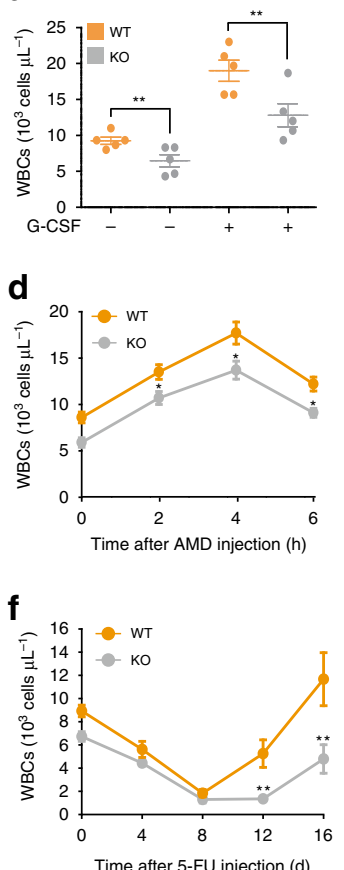

b

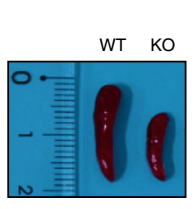

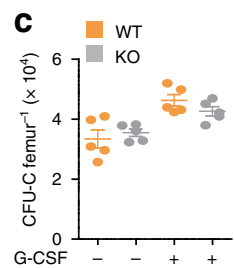

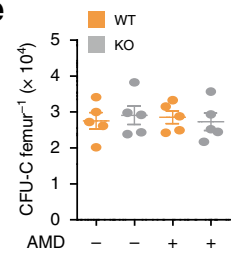

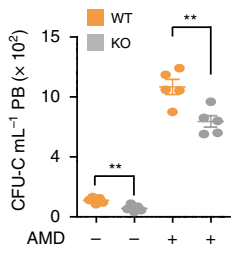

g

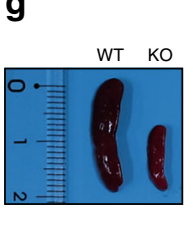

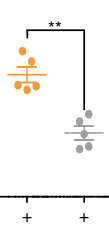
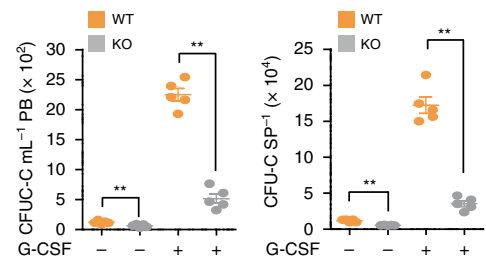

Fig. 2 Impaired mobilization of KO HSPCs from the BM into the periphery. a Comparison of WBC counts between WT and KO mice on day 5 after G-CSF treatment. $n=5$. $\mathbf{b}$ Representative image of spleens from WT and KO mice (left) and absolute numbers of splenocytes (right) for WT and KO mice on day 5 after G-CSF treatment. $n=5$. c CFU-C in the BM (femur), PB, and spleen (SP) of WT and KO mice on day 5 after G-CSF treatment. $n=5$. d Comparison of WBC counts between WT and KO mice $3 \mathrm{~h}$ after AMD3100 treatment. $n=5$. e CFU-C in the BM (femur) and PB of WT and KO mice $3 \mathrm{~h}$ after AMD3100 treatment. $n=5$. f Comparison of WBC counts between WT and KO mice at indicated time points after 5 -FU treatment. $n=5$. $\mathbf{g}$ Representative image of the spleen from a WT or KO mouse (left) and absolute number of splenocytes (right) for WT or KO mice at indicated time points after 5-FU treatment. $n=5$. h CFU-C in the BM (femur), PB, and spleen (SP) of WT and KO mice at indicated time points after 5-FU treatment. $n=5$. " $-{ }^{\prime \prime}$, before treatment with G-CSF or AMD3100; " + ", after treatment with G-CSF or AMD3100. Statistical significance was assessed by two-tailed Student's $t$-test. ${ }^{\star \star} P<0.01$. All data are presented as the mean \pm SEM. Source data are provided as a Source Data File
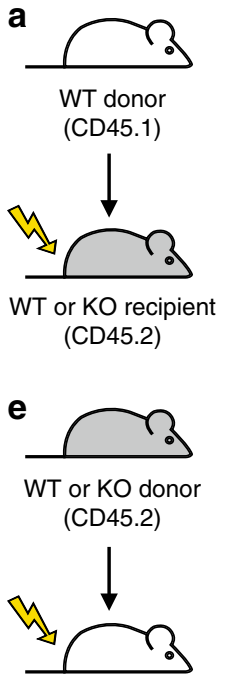

WT recipient (CD45.1) b

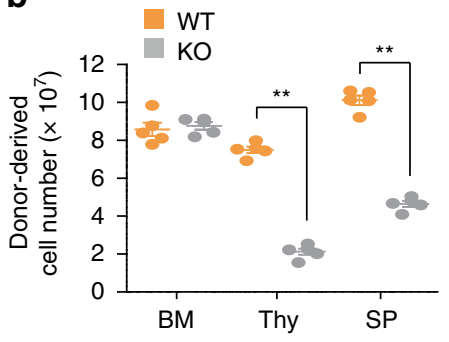

f

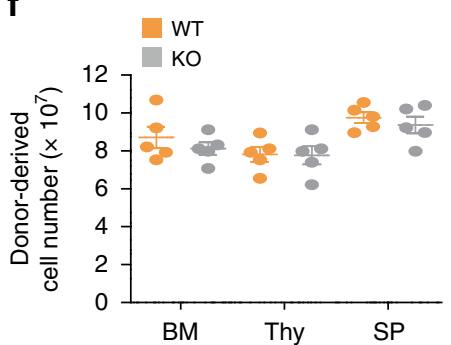

C

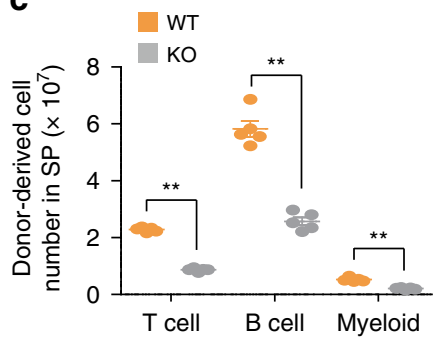

g

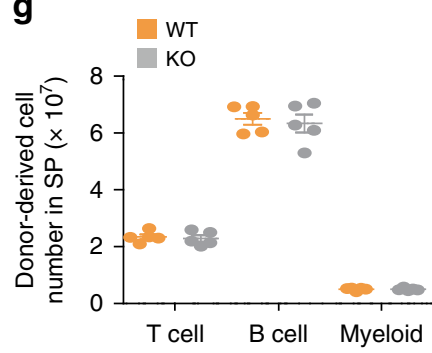

d

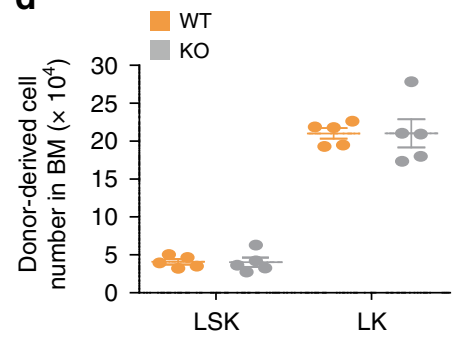

h

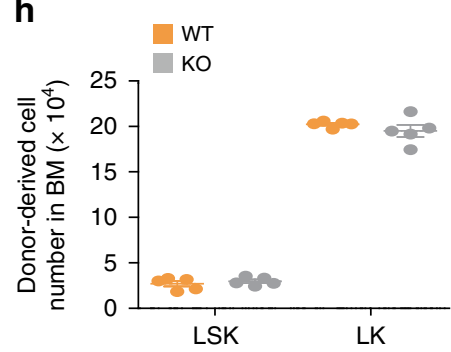

Fig. 3 An extrinsic factor from BMSCs is responsible for the impaired mobilization of KO HSPCs from the BM into the periphery. a-d Lethally irradiated WT and KO CD45.2 mice were transplanted with LSK cells from WT CD45.1 mice. $n=5$ per group. a Schematic representation of LSK cell adoptive transfer. b Absolute number of donor-derived cells in the BM, thymus (Thy), and spleen (SP) of recipients. c Absolute number of donor-derived T cells, B cells, and myeloid cells (Myeloid) in the spleen of recipients. d Absolute number of donor-derived LSK cells (LSK) and LK cells (LK) in the BM of recipients. e- $\mathbf{h}$ Lethally irradiated WT CD45.1 mice were transplanted with LSK cells from WT or KO CD45.2 mice. $n=5$ per group. e Schematic representation of LSK cell adoptive transfer. $\mathbf{f}$ Absolute numbers of donor-derived cells in the BM, Thy, and SP of recipients. $\mathbf{g}$ Absolute numbers of donor-derived T cells, B cells, and myeloid cells in the spleen of recipients. $\mathbf{h}$ Absolute numbers of donor-derived LSK and LK cells in the BM of recipients. Statistical significance was assessed by two-tailed Student's $t$-test. ${ }^{\star} P<0.01$. All data are presented as the mean \pm SEM. Source data are provided as a Source Data File 

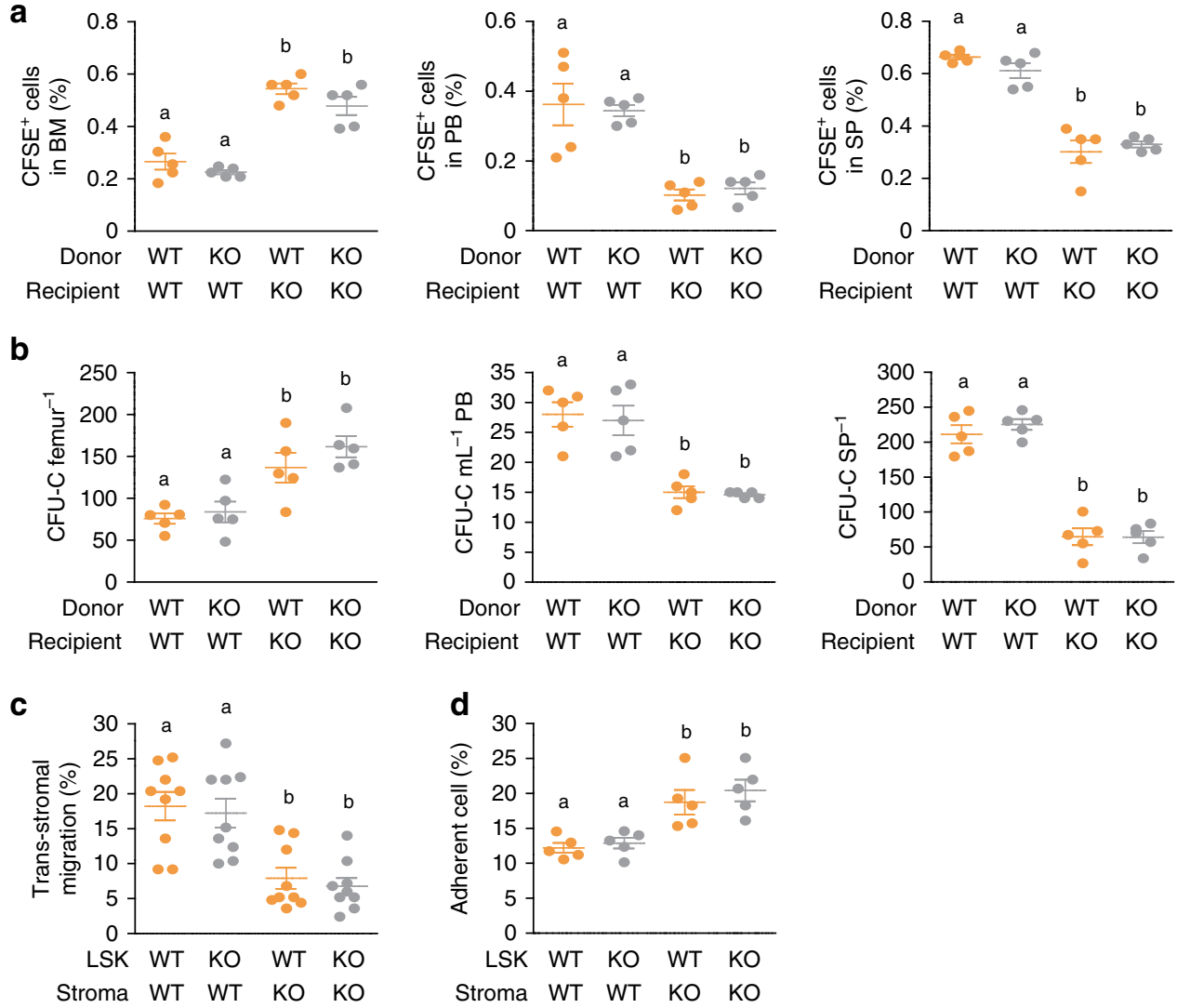

Fig. 4 Phc2 deficiency leads to impaired HSPC mobilization from the BM into the periphery via stronger adhesion between HSPCs and BMSCs in BM niches. a, b CFSE-labeled LSK cells from WT and KO mice were intravenously injected into lethally irradiated recipient mice. $n=5$ per group. $\mathbf{a}$ The frequencies of $\mathrm{CFSE}^{+}$donor cells were measured in the BM, PB and spleen (SP) of recipients at $16 \mathrm{~h}$ after injection. $\mathbf{b}$ The frequencies of donor-derived clonogenic progenitors (CFU-C) homing to the BM (femur), PB and spleen (SP) of recipients at $16 \mathrm{~h}$ after injection. c Relative ratio of migrated WT or KO LSK cells (LSK) through WT or KO BMSCs (Stroma). $n=9$ per group. d Relative ratio of adhered WT or KO LSK to WT or KO stroma. $n=5$ per group. Statistical significance was assessed by one-way ANOVA with Tukey HSD analysis. Mean values not sharing the same superscript letter ( ${ }^{a}$, b) differ significantly at $P<0.05$. All data are presented as the mean \pm SEM. Source data are provided as a Source Data File

putative Phc2 binding sites located in the Vcam1 gene (Fig. 6a). This analysis revealed the strong binding of Phc2 to the promoter region of the Vcam1 locus (Fig. 6b, primers \#2 and \#3). Interestingly, direct binding by other members of the canonical PRC1 family, such as Bmil, Ring1b, and Cbx7, was also found around the same region covered by primers \#2 and \#3 (Fig. 6c). Consistent with this, Bmil, Ring1b, and Cbx7 binding to these regions were significantly decreased in the absence of Phc2 (Fig. 6c). The acetylation of histone $\mathrm{H} 3$ at lysine 9/14 (H3K9ac), trimethylation of histone $\mathrm{H} 3$ at lysine 4 (H3K4me3), H3K27me3, and H2AK119ub were observed around the same region covered by primers \#2 and \#3 (Fig. 6c). H3K9ac and H3K4me3 were increased, whereas $\mathrm{H} 3 \mathrm{~K} 27 \mathrm{me} 3$ and H2AK119ub were decreased in KO BM cells compared to WT BM cells (Fig. 6c). Furthermore, the treatment of BMSCs (OP9 cells) with GSK126, an Ezh2 inhibitor $^{25}$, led to the upregulation of Vcaml expression in a dose-dependent manner (Fig. 6d, e and Supplementary Fig. 11). Consistent with these observations, the binding of Phc2, Bmil, and Ring $1 \mathrm{~b}$ to the regions covered by primers \#2 and \#3 was decreased in GSK126-treated BMSCs (OP9 cells) (Fig. 6f).

Next, we then asked whether GSK126 treatment could mimic Phc2 deficiency in vivo. Different dose of GSK126 was administered intraperitoneally into WT mice. Sixteen hours after GSK injection, BMSCs were isolated and analyzed for VCAM-1 expression. We also measured the capacity of WT LSK cells to transmigrate across their GSK126-treated BMSCs using a trans-stromal migration assay. Results clearly showed that GSK126 treatment increased both VCAM-1 expression in BMSCs and adherence of LSKs onto BMSCs in a dose-dependent manner (Supplementary Fig. 12). Therefore, the canonical PRC1 containing Phc2 can repress Vcam1 expression through histone modifications, such as by enhancing H3K27me3 and H2AK119ub, in BMSCs.

Anti-VCAM-1 Ab treatment restores $\mathrm{Phc}^{-/-}$HSPC mobilization. Next, we examined whether blocking the interaction between VCAM-1 and VLA-4 could restore HSPC mobilization in $\mathrm{KO}$ mice. We first attempted to measure the mobilization capacity of WT LSK cells across KO BMSCs after treatment with neutralizing antibodies (Abs) against VCAM-1 and/or VLA-4. Treatment with either $\mathrm{Ab}$ restored the trans-stromal migration activity of LSK cells across KO BMSCs to a level similar to that of LSK cells across WT BMSCs (Fig. 7a). Additionally, there was no significant difference in the relative ratio of LSK cell adhesion to KO or WT BMSCs after treatment with the neutralizing Abs (Fig. 7b). The in vivo homing assay further confirmed that blocking the interaction between VCAM-1 and VLA-4 could restore LSK cell mobilization from the $\mathrm{KO} B M$ into the $\mathrm{PB}$ and spleen (Fig. 7c, d and Supplementary Fig. 13). We then tested whether treatment of the neutralizing VCAM-1 Ab could rescue steady-state HSPC mobilization in KO mice. Results clearly demonstrated that the administration of the neutralizing VCAM- 
a
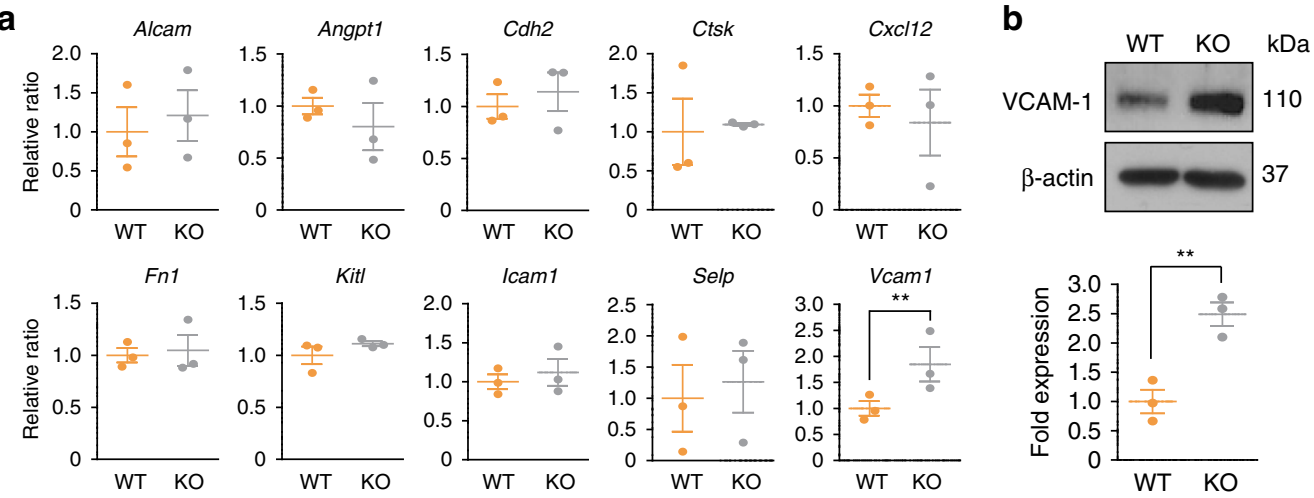

C

d
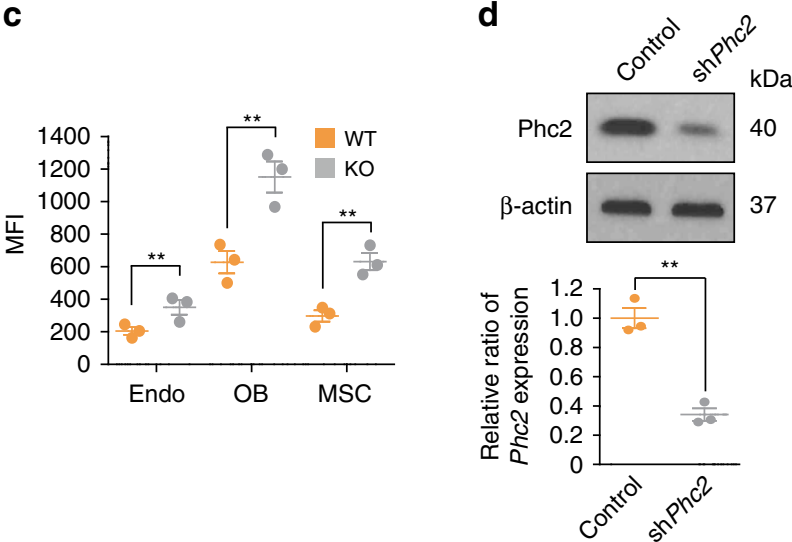

e

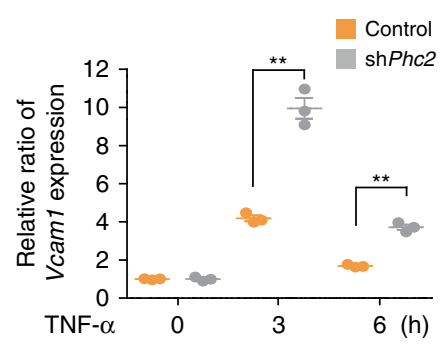

$\mathbf{f}$
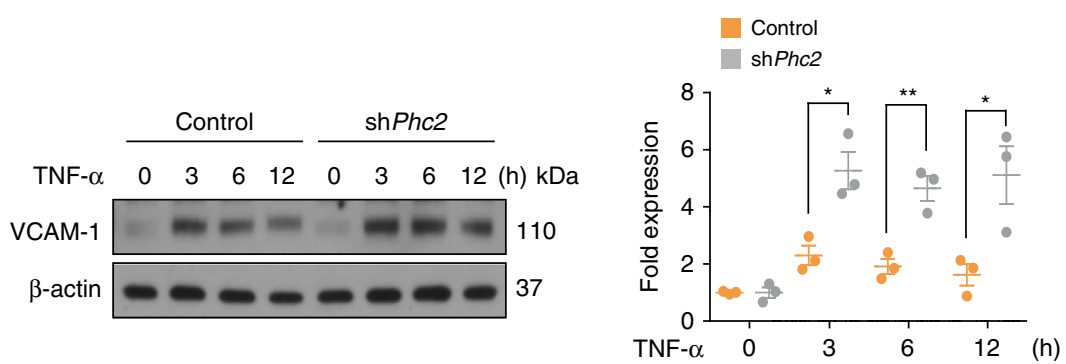

Fig. 5 Phc2 inhibits VCAM-1 expression in BMSCs. a mRNA expression patterns of genes related to HSPC mobilization in BM niches from WT and KO mice were accessed by qRT-PCR. $n=3$. b Lysates from WT and KO BMSCs were immunoblotted to analyze VCAM-1 expression. $n=3$. $\mathbf{c}$ Mean fluorescence intensity (MFI) of VCAM-1 expression in BMSCs from WT and KO mice analyzed by flow cytometry. $n=3$ Endo, endothelial cells; OB, osteoblasts; MSC, mesenchymal stem cells. d Control plasmid (Control) and shPhc2 plasmid (shPhc2) were transfected into BMSCs (OP9 cells). qRT-PCR (bottom) and immunoblot (top) analyses were then performed to measure the Phc2 expression levels. $n=3$. e, $\mathbf{f}$ Control transfectants (Control) and shPhc2 transfectants (shPhc2) were treated with TNF- $\alpha$ for the indicated time period. GRT-PCR (e) and immunoblot (f) analyses were then performed to measure VCAM-1 expression. Relative fold change of each Vcam1 mRNA expression from Control or shPhc2 was calculated relative to the basal level of that in unstimulated Control or shPhc2, respectively. $n=3$. Statistical significance was assessed by two-tailed Student's $t$-test. ${ }^{\star} P<0.05 ;{ }^{\star \star} P<0.01$. All data are presented as the mean \pm SEM. Source data are provided as a Source Data File

$1 \mathrm{Ab}$ in KO mice successfully reconstitutes HSPC mobilization from BM into periphery (Fig. $7 \mathrm{e}-\mathrm{g}$ ).

We also performed a G-CSF-, AMD3100-, or 5-FU-induced LSK cell mobilization assay using WT and KO mice treated with the neutralizing VCAM-1 Ab. There were no significant differences in the absolute numbers of WBCs and splenocytes between the WT and KO mice treated with anti-VCAM-1 on day 5 after the G-CSF treatment and on day 16 following the 5-FU challenge (Fig. 8a, b, f, g). Similarly, the absolute number of LSK cells and the clonogenic progenitor frequency in the $\mathrm{BM}, \mathrm{PB}$, and spleen were comparable between WT and $\mathrm{KO}$ recipients treated with the neutralizing $\mathrm{Ab}$ on day 5 after the G-CSF treatment and on day 16 following the 5-FU challenge (Fig. 8c, h, Supplementary Figs. 14 and 15). Consistent with these results, the defective
HSPC migration into the PB of the KO mice was reversed by treating the mice with anti-VCAM-1, as observed in the HSPC mobilization assay using AMD3100 alone or in combination with G-CSF (Fig. 8d, e and Supplementary Fig. 16).

To further reinforce these observations, we assessed the significance of VCAM-1 blockage when the migration defect of HSPCs are driven by extrinsic microenvironment which mimics the phenotype associated with Phc2 KO mice. First, we adoptively transferred LSK cells isolated from WT CD45.1 mice into irradiated WT or KO CD45.2 recipient mice and then performed the same G-CSF-induced LSK cell mobilization assay using recipient mice treated with the neutralizing VCAM-1 Ab (Supplementary Fig. 17). Consistent to the HSPC mobilization assay in KO mice, WT cells reconstituted in irradiated $\mathrm{KO}$ 

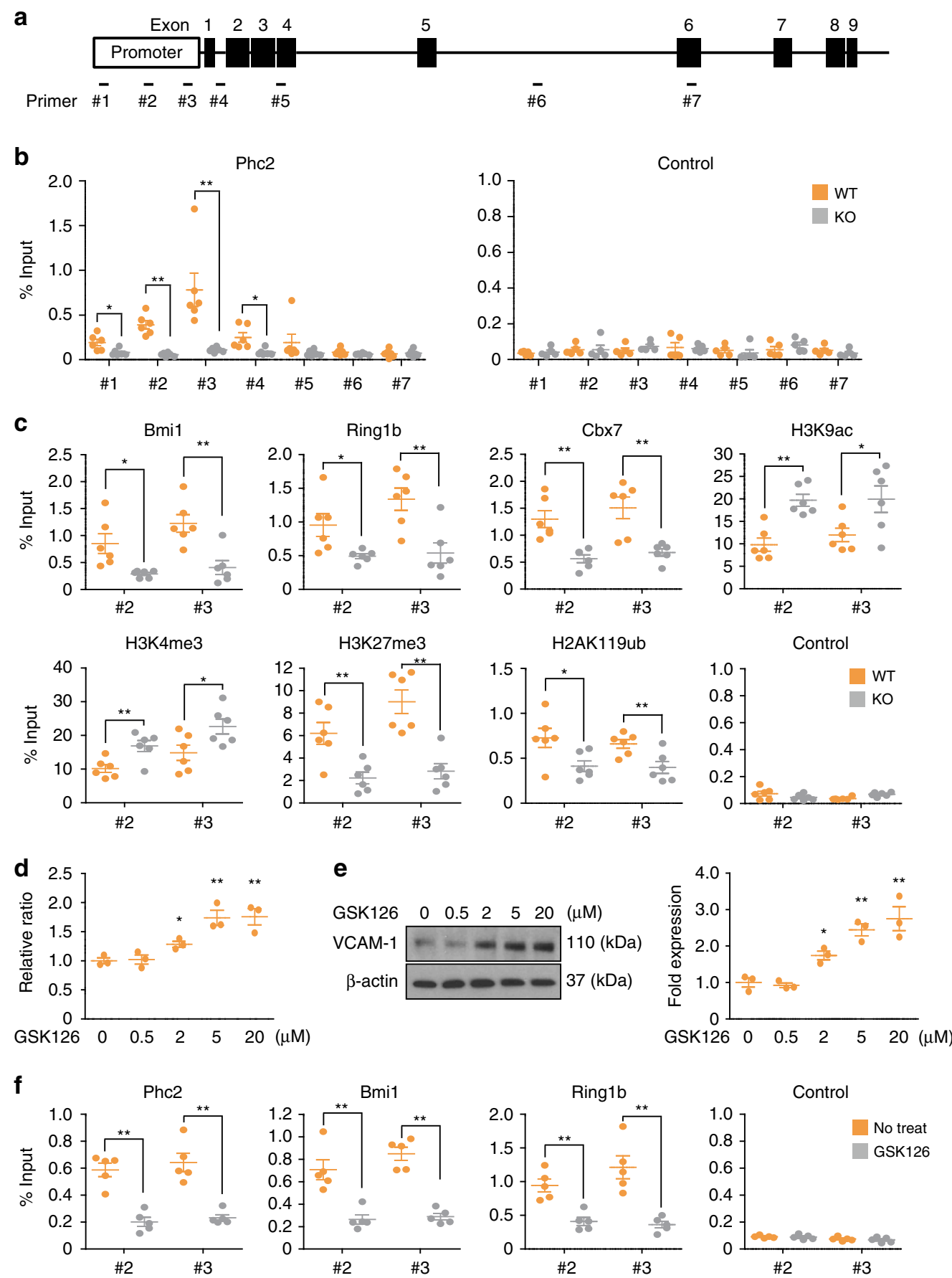

Fig. 6 Phc2 binds to the Vcam1 gene locus and suppresses Vcam1 gene expression through histone modifications. a Schematic representation of the Vcam 1 gene locus. The locations of primers \#1 to \#7 and exons are indicated. b ChIP was performed with WT and KO BM cell lysates using anti-Phc2 Ab (Phc2) or a control Ab (Control). DNA recovered from ChIP was then analyzed by qPCR. $n=5$. c ChIP was performed using the same samples with indicated Abs. DNA recovered from ChIP was then analyzed by GPCR. $n=6$. Control, ChIP using each isotype control Ab. d, e BMSCs (OP9 cells) were treated with various concentrations of GSK126 for $48 \mathrm{~h}$. qRT-PCR (d) and immunoblot (e) analyses were then performed to measure the VCAM-1 expression levels. $n$ =3. $\mathbf{f}$ BMSCs (OP9 cells) were treated with GSK126 $(5 \mu \mathrm{M})$ for $48 \mathrm{~h}$. ChIP was performed using cell lysates with indicated Abs. DNA recovered from ChIP was then analyzed by qPCR. $n=5$. Control, ChIP using each isotype control Ab. Statistical significance was assessed by two-tailed Student's $t$-test. ${ }^{\star} P<$ $0.05 ;{ }^{\star \star} P<0.01$. All data are presented as the mean \pm SEM. Source data are provided as a Source Data File

recipient cells were only partially rescued the mobilization defects when the single G-CSF agent was treated (Supplementary Fig. 17). However, the administration of the neutralizing VCAM-1 Ab in combination with $\mathrm{G}-\mathrm{CSF}$ in $\mathrm{KO}$ recipient mice completely reconstituted WT donor HSPC mobilization from BM into periphery, the same degree to WT recipient mice (Supplementary Fig. 17). Taken together, these results demonstrate that blocking the interaction between VCAM-1 and VLA-4 can restore HSPC migration from the $\mathrm{BM}$ into the periphery of $\mathrm{KO}$ mice.

\section{Discussion}

In this study, we demonstrated that Phc2 binds to the Vcam1 locus to repress its transcription by enhancing $\mathrm{H} 3 \mathrm{~K} 27 \mathrm{me} 3$ and 
a

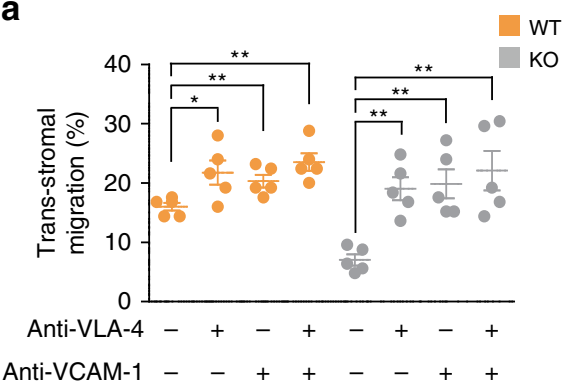

b

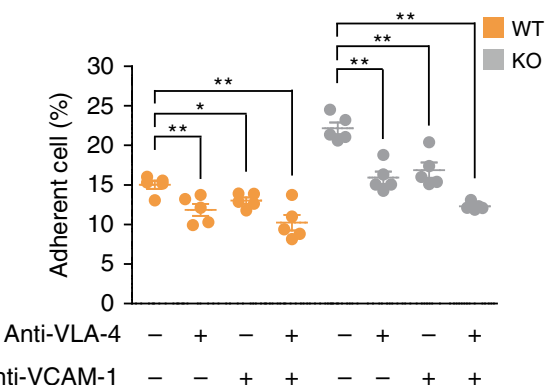

C
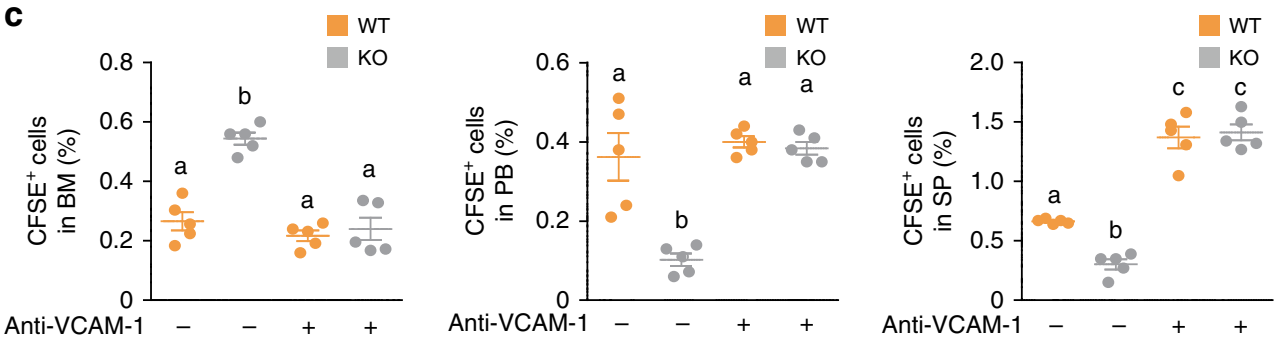

d

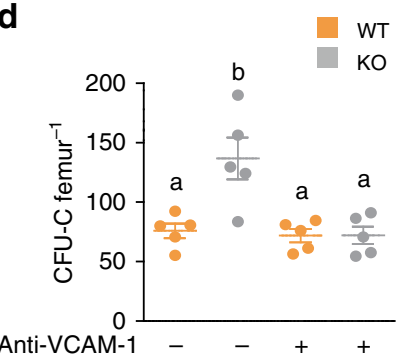

e

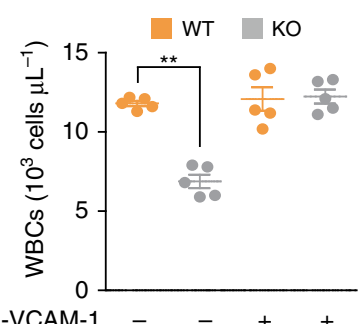

g

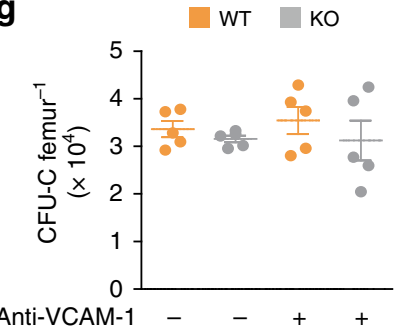

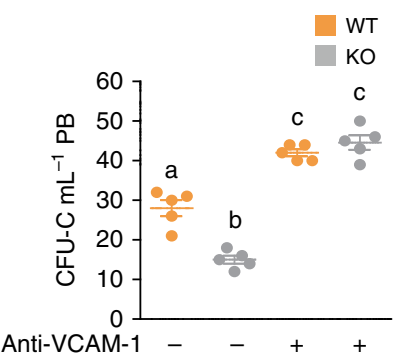

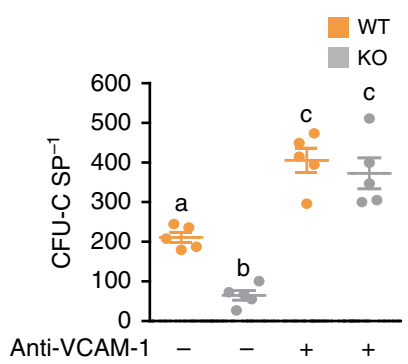

f
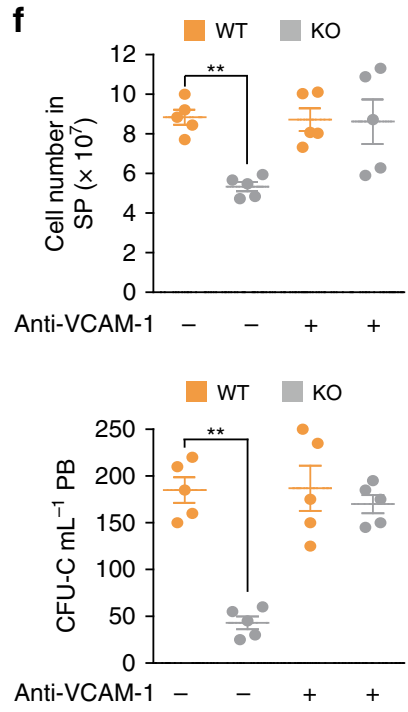

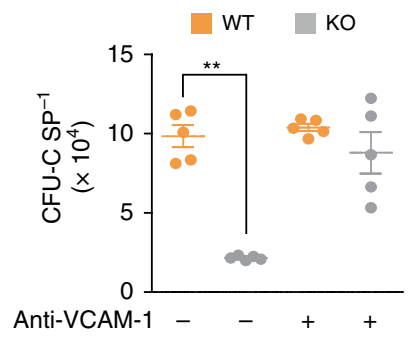

H2AK119ub in BMSCs. In the absence of Phc2, the increased Vcam1 expression in BMSCs strengthened the interaction between HSPCs and BMSCs, compromising timely HSPC mobilization from the BM into the periphery, which in turn led to a systemic immunodeficiency. Consistent with these observations, treatment with a neutralizing $\mathrm{Ab}$ against VCAM-1 in Phc2deficient mice restored HSPC mobilization from the BM into the periphery. These data firmly establish a causative role for the epigenetic regulation of VCAM-1 in regulating HSPC mobilization, as a key downstream mediator of Phc2 functions.

Recent genetic evidence from conditional VCAM-1- or VLA-4deficient mice further supports our model of the VLA-4/VCAM-1 pathway as downstream of $\mathrm{Phc} 2$ function, and as expected from our results with Phc2-deficient mice overexpressing VCAM-1, VCAM-1- or VLA-4 deficient mice exhibit increased circulating progenitors and immature B cells, and impaired HSPC homing to $\mathrm{BM}^{26-30}$. The BM homing defect of HSPCs in these mice became obvious when the mice were treated with $5-\mathrm{FU}$ or G-CSF 26,27 . However, the Phc2-deficient mice still displayed the migration defect of HSPCs from the BM into the periphery after treatment with these mobilization agents.

The identification of a single key mediator of Phc2 function may have important clinical implications. Similar to previous observations with conditional VCAM-1- or VLA-4-deficient 
Fig. 7 Blocking the interaction between VCAM-1 and VLA-4 restores steady-state HSPC mobilization in KO mice. a Relative ratio of migrated WT LSK cells (LSK) through WT or KO BMSCs in the presence of anti-VLA-4 Ab and/or anti-VCAM-1 Ab. $n=5$ per group. Statistical significance was assessed by twotailed Student's $t$-test. ${ }^{\star} P<0.05$; ${ }^{\star \star} P<0.01$. b Relative ratio of adhered WT or KO LSK to KO BMSCs in the presence of anti-VLA-4 Ab and/or anti-VCAM$1 \mathrm{Ab} . n=5$ per group. Statistical significance was assessed by two-tailed Student's $t$-test. ${ }^{\star} P<0.05 ;{ }^{\star \star} P<0.01$. c, $\mathbf{d}$ CFSE-labeled LSK cells from WT mice were intravenously injected into lethally irradiated WT or KO mice. Anti-VCAM-1 Ab or its isotype control Ab $\left(2 \mathrm{mg} \mathrm{kg}^{-1}\right)$ was intravenously injected into WT and $\mathrm{KO}$ recipient mice $1 \mathrm{~h}$ before adoptive cell transfer. $n=5$ per group. $\mathbf{c}$ The frequencies of $\mathrm{CFSE}^{+}$donor cells were measured in the BM, $\mathrm{PB}$, and, spleen (SP) of recipients at $16 \mathrm{~h}$ after adoptive cell transfer. $\mathbf{d}$ The frequencies of donor-derived clonogenic progenitors (CFU-C) homing to the BM (femur), PB and SP of recipients at $16 \mathrm{~h}$ after adoptive cell transfer. Statistical significance was assessed by one-way ANOVA with Tukey HSD analysis. Mean values not sharing the same superscript letter $(a, b, c)$ differ significantly at $P<0.05$. e-g Anti-VCAM-1 Ab or its isotype control Ab $\left(2 \mathrm{mg} \mathrm{kg}^{-1}\right)$ was intravenously injected into WT and KO mice for 3 days. $n=5$ per group. e Comparison of WBC counts between WT and KO mice on day 3 after Ab treatment.

f Absolute number of splenocytes for WT and KO mice on day 3 after Ab treatment. $\mathbf{g}$ CFU-C in the BM (femur), PB, and SP of WT and KO mice on day 3 after $\mathrm{Ab}$ treatment. Statistical significance was assessed by two-tailed Student's $t$-test. ${ }^{\star \star} P<0.01$. All data are presented as the mean \pm SEM. WT, WT recipient mice; KO, KO recipient mice. "-", treatment with an isotype control Ab; " + ", treatment with anti-VCAM-1 Ab. Source data are provided as a Source Data File

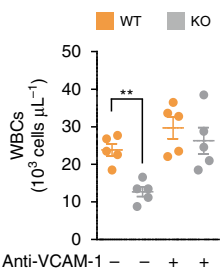

d

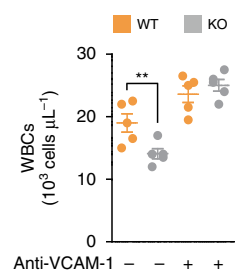

$\mathbf{f}$

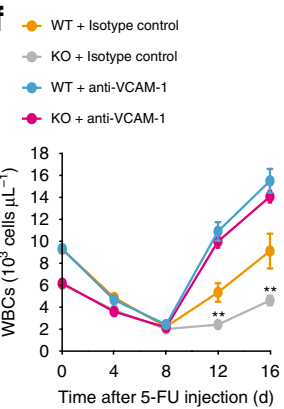

Time after 5-FU injection (d) b

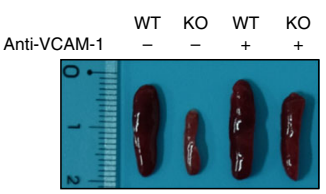

e

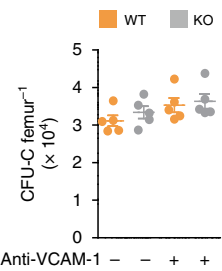

g

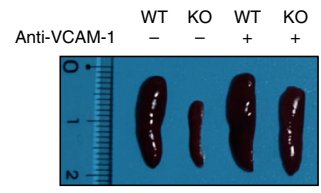

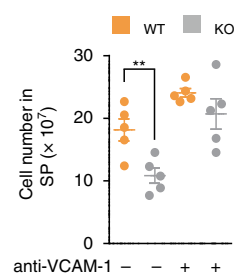
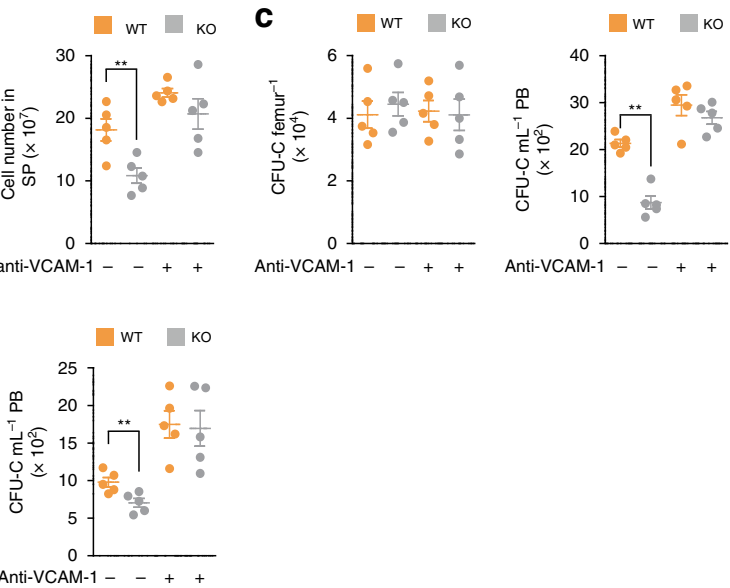

Anti-VCAM-1 - + +

h

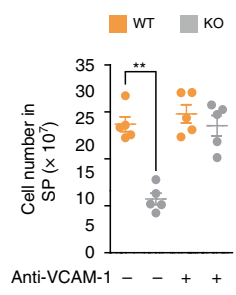

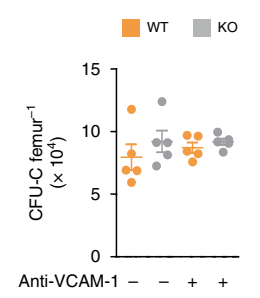

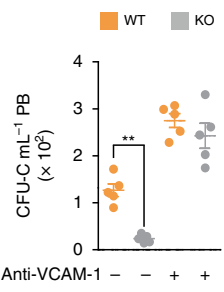

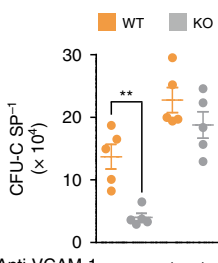

Anti-VCAM-1 - + +

Fig. 8 Neutralizing interaction between VCAM-1 and VLA-4 reverses the defect involving regimen-induced HSPC mobilization in KO mice. a-c G-CSFinduced HSPC mobilization assays with anti-VCAM-1 Ab. $n=5$ per group. a WBC counts from experimental animals until day 5 after G-CSF and Ab treatment. $\mathbf{b}$ Representative image of spleens (left) and absolute number of splenocytes from experimental animals on day 5 after $\mathrm{G}$-CSF and Ab treatment (right). c CFU-C in the BM (femur), PB, and spleen (SP) from experimental animals on day 5 after G-CSF and Ab treatment. d, e WBC counts (d) and CFU$\mathrm{C}(\mathbf{e})$ in PB from experimental animals $3 \mathrm{~h}$ after AMD3100 and Ab treatment. $n=5$ per group. $\mathbf{f}-\mathbf{h}$ 5-FU-induced HSPC cell mobilization assays with antiVCAM-1 Ab. $n=5$ per group. $\mathbf{f}$ Comparison of WBC counts between WT and KO mice at indicated time points after 5-FU and Ab treatment.

g Representative image of spleen from a WT and KO mouse (left) and absolute number of splenocytes (right) of WT and KO mice on day 16 after 5-FU and Ab treatment. $\mathbf{h}$ CFU-C in the BM (femur), PB, and spleen (SP) of WT and KO mice on day 16 after 5-FU and Ab treatment. "-", treatment with an isotype control Ab; " + ", treatment with anti-VCAM-1 Ab. Statistical significance was assessed by two-tailed Student's $t$-test. ${ }^{\star \star} P<0.01$. All data are presented as the mean \pm SEM. Source data are provided as a Source Data File

mice ${ }^{26-30}$, we found an abnormality of only steady-state HSPC mobilization in Phc2-deficient mice. Additionally, treatment with the anti-VCAM-1 Ab or anti-VLA-4 Ab in primates or mice does not cause any other physiological change ${ }^{31,32}$. Combined evidence may suggest a dominant role for VCAM-1 in steady-state HSPC migration due to the VCAM-1 expression pattern. In steady-state conditions, the BMSC is the only cell type that constitutively expresses VCAM-1 at physiological levels, although vascular endothelial cells and other immune cell populations express minimally detectable levels of VCAM-1 ${ }^{33-38}$. Furthermore, with a proof-of-concept experiment, we successfully demonstrated that blocking VCAM-1 in BM niches critically reverses the HSPC mobilization defect in Phc2-deficient mice. Therefore, along with other recent data ${ }^{39-43}$, our results suggest that modulating the interaction between VCAM- 1 and VLA-4 in $\mathrm{BM}$ niches provides potential strategies to develop HSPC mobilizing agents or anti-leukemia drugs. Therefore, it will be interesting to observe whether a specific inhibitor that blocks Phc2 
function is useful for keeping malignant cells from hematopoietic lineages within the BM.

To our knowledge, we are the first to provide evidence that a systemic immunodeficiency can be caused by a defect in a particular epigenetic regulator that represses the expression of a certain cell adhesion molecule in BMSCs. Our study also demonstrates the molecular mechanism through which the epigenetic regulation of extrinsic factors can help finely tune the interaction between HSPCs and BMSCs within BM niches during HSPC mobilization. Although several genes that are involved in hematopoiesis have been identified as targets for epigenetic regulation, the epigenetic effect on HSPC mobilization has not been elucidated. Notably, the vast majority of previously identified genes play important roles in defining the intrinsic property of HSCs and the development of leukemia $8,44,45$.

Like deficiencies in other epigenetic regulators, a single PcG gene deficiency in mice mostly causes early embryonic lethality or a limited life span with severe developmental defects, including an intrinsic defect in $\mathrm{HSCs}^{8,46,47}$. This characteristic hinders the search for additional roles of polycomb proteins in the regulation of immune phenomena aside from their known functions in HSC differentiation. Similar to other members of the PcG protein family, Phc2 is expressed in various tissues and cells and regulates the transcription of $C d k n 2 a\left(p 16^{I N K 4 a}\right.$ and $\left.p 19^{A R F}\right)$ and hox genes through direct associations with chromatin ${ }^{8,14}$. However, Phc2deficient mice are fertile and exhibit less severe defects associated with the abnormal axial skeleton and Peyer's patch ${ }^{14,48}$. Moreover, the systemic immunodeficiency observed in Phc2-deficient mice is not caused by an intrinsic defect in HSC function, but instead, the Phc2 deficiency causes a defect in HSPC mobilization by enhancing the expression of VCAM-1 in BMSCs. This may suggest that most of the Phc2 functions in mammals are dispensable or can be replaced by other components of the canonical PRC1 due to a structural heterogeneity of PRC1 or functional redundancy of $\mathrm{PcG}$ proteins. In fact, each of the mammalian PRC2 or PRC1 subunits has several paralogs ${ }^{8-13}$. For example, $\mathrm{Phc} 2$ has two paralogs, $\mathrm{Phc1}$ and $\mathrm{Phc} 3^{8-13}$. Previous observation revealed that Phc1 deficiency causes a defect in HSC's selfrenewal activity 49,50 . Therefore, $\mathrm{Phc1}$, but not phc2, might be critical for HSC maintenance and function. Consistent with this idea, the expression and epigenetic pattern of Cdkn2a ( $p 16^{I N K 4 a}$ and $p 19^{A R F}$ ) in KO BM cells showed no significant differences compared to those in WT BM cells (Supplementary Fig. 18). Another example of nonredundant function by each paralog within PRC2 or PRC1 components might be found in Ezh proteins or Cbx proteins, respectively ${ }^{51-55}$. Among Ezh proteins, Ezh1 is critical for self-renewal activity of HSCs, whereas Ezh2 is dispensable for proper HSC function ${ }^{51,52}$. Also, Cbx7 is the only paralog which is necessary for self-renewal activity of HSC among Cbx proteins ${ }^{53,54}$.

In our molecular model, PRC2 containing Ezh2 with methyltransferase activity initiates $\mathrm{H} 3 \mathrm{~K} 27 \mathrm{me} 3$ on the Vcam1 locus in BMSCs because the Ezh2 inhibitor (GSK126) treatment of BMSCs increases the Vcam1 mRNA levels by negating the recognition of $\mathrm{H} 3 \mathrm{~K} 27 \mathrm{me} 3$ by the canonical PRC1 complex containing Phc2 on the same locus. The canonical PRC1 then recognizes and binds to $\mathrm{H} 3 \mathrm{~K} 27 \mathrm{me} 3$ to maintain Vcam 1 gene suppression. The recognition of $\mathrm{H} 3 \mathrm{~K} 27 \mathrm{me} 3$ on the Vcam 1 locus by the canonical PRC1 is dependent on Phc2 expression. Consistently, we observed reductions in the binding of other canonical PRC1 components to the Vcam1 locus when Phc2 was not expressed. Our study also revealed that the H2AK119ub activity of the canonical PRC1 on the Vcam1 locus was dependent on $P h c 2$ expression, which is consistent with a recent observation that the interaction between Bmil and Phc2 is critical for the $\mathrm{H} 2 \mathrm{AK} 119 \mathrm{ub}$ activity of the canonical PRC1 ${ }^{55}$.
In conclusion, the results of our study demonstrated Phc2regulated HSPC mobilization through the direct repression of Vcam1 gene expression in BMSCs. These findings may offer a novel approach to manipulating the epigenetic patterns of genes involved in HSPC mobilization, leading to new therapeutic strategies for BM transplantation and leukemia treatment.

\section{Methods}

Experimental mice and phenotypic analysis. $\mathrm{Phc2^{+/- }}(\mathrm{Phc2}$ heterozygote) mice with a C57BL/6 background were used in this study ${ }^{14}$. C57BL/6 and B6 CD45.1 mice were obtained from The Jackson Laboratory. WT and $\mathrm{KO}$ mice were bred from $P h c 2^{+/-}$mice. All animals received proper care in accordance with the National Institutes of Health Guide for the Care and Use of Laboratory Animals. The study protocol was approved by the Institutional Animal Care and Use Committee of Korea University (protocol numbers: KUIACUC-20110104-3, KUIACUC-20141002-4, and KUIACUC-20160927-3).

Cell culture. BMSCs were obtained and maintained from WT and $\mathrm{KO}$ mice ${ }^{56}$ Briefly, bone marrow (BM) cells were isolated from the femurs and tibias of 8-week-old mice and cultured in Dulbecco's modified Eagle medium (Welgene LM 001-05) supplemented with 10\% fetal bovine serum (FBS; Welgene S 001-01), $10 \%$ horse serum (Welgene S 104-01), 2 mM L-glutamine (Welgene LS 002-01), $2 \mathrm{mM}$ sodium pyruvate (Welgene LS $002-02$ ), and $100 \mathrm{U} \mathrm{ml}^{-1}$ penicillin/streptomycin (Welgene LS 202-02) at $37^{\circ} \mathrm{C}$ and $5 \% \mathrm{CO}_{2}$. Non-adherent cells were removed by washing with PBS after overnight incubation. Adherent cells were cultured and fresh medium was added every 2 days. After 4 weeks of culture, cells were harvested by incubation with trypsin/EDTA (Welgene LS 015-01) for $2 \mathrm{~min}$ at $37^{\circ} \mathrm{C}$ and plated onto culture dishes. At two weeks after the first passage, cells were harvested and cultured under the same conditions for subsequent passages (passages 2-7). OP9 BMSC line (ATCC CRL-2749) was maintained in alpha MEM (Welgene LM 008-02) supplemented with $20 \%$ FBS and $1 \%$ penicillin/streptomycin at $37^{\circ} \mathrm{C}$ and $5 \% \mathrm{CO}_{2}$. For induction of VCAM-1, OP9 cells were treated with TNFa (100 ng ml ${ }^{-1}$; R\&D Systems 410-MT) for the indicated time period. To inhibit Ezh activity, OP9 cells were treated with various concentrations of GSK126, an Ezh2 inhibitor (Xcessbio Biosciences M60071-2), for $48 \mathrm{~h}$.

PB count, flow cytometry analysis, and cell sorting. PB samples from each mouse were collected by retro-orbital bleeding and analyzed using a Coulter LH 780 Hematology Analyzer (Beckman Coulter). For flow cytometry, single cell suspensions were prepared from the BM, $\mathrm{PB}$, thymus, and spleen of each mouse. After removing red blood cells, cells were stained with MACS buffer at $4{ }^{\circ} \mathrm{C}$ in the presence of Fc Block Ab (BD Biosciences 553141, dilution 1:100). Abs purchased from BD Biosciences, Biolegend or Thermo Fisher Scientific were used to detect the following cell surface markers by flow cytometry: B220 (BD Biosciences 553090, dilution 1:100), CD3ع (Thermo Fisher Scientific 11-0031-63, dilution 1:100), CD4 (BD Biosciences 553729, dilution 1:100), CD8 (BD Biosciences 553030, dilution 1:100), CD11b (Thermo Fisher Scientific 11-0112-41, dilution 1:100), CD11c (BD Biosciences 553802, dilution 1:100), CD16:32 (Thermo Fisher Scientific 14-016181, dilution 1:100), CD25 (Thermo Fisher Scientific 17-0251-81, dilution 1:100), CD31 (BD Biosciences 561814, dilution 1:100), CD34 (BD Biosciences 560238, dilution 1:100), CD44 (BD Biosciences 561861, dilution 1:100), CD45 (BD Biosciences 550539, dilution 1:100), CD48 (Biolegend 103412, dilution 1:100), CD51 (BD Biosciences 551187, dilution 1:100), CD106 (BD Biosciences 553332, dilution 1:100), CD127 (Thermo Fisher Scientific 45-1271-80, dilution 1:100), CD150 (Biolegend 115922, dilution 1:100), c-kit (Thermo Fisher Scientific 17-1171-81, dilution 1:100), F4/80 (Thermo Fisher Scientific MF48004, dilution 1:100), Flt3 (Thermo Fisher Scientific 46-1351-80, dilution 1:100), IgD (BD Biosciences 562022, dilution 1:100), IgM (BD Biosciences 550676, dilution 1:100), NK1.1 (BD Biosciences 557391, dilution 1:100), and Sca-1 (BD Biosciences 558162, dilution 1:100). After washing several times with PBS, stained cells were resuspended in PBS and analyzed by flow cytometry using a FACSCalibur with CellQuest software (BD Biosciences). Analysis and sorting of HSPCs (Lin ${ }^{-} \mathrm{Sca}-1^{+} \mathrm{c}-\mathrm{kit}^{+}$cells; LSK cells) and early progenitor cells were performed using MACS cell separation system and FACSAria Fusion Cell Sorter (BD Biosciences) ${ }^{57}$. Depletion of specific lineage cells was achieved with a lineage-specific Ab cocktail and anti-biotin microbeads (Miltenyi Biotec 130-090-858, dilution 1:5). Gating strategy for LSK cell sorting ${ }^{58}$ was depicted in Supplementary Fig. 19.

Histological analysis and immunoblotting. For histological analysis ${ }^{59}$, tissue samples were fixed with $10 \%$ formalin (Sigma, HT5011) and embedded in paraffin. Then, sections measuring $5 \mu \mathrm{m}$ were cut using a Leica CM1800 cryostat (Leica Microsystems), and air dried at room temperature. After dry, slides containing each tissue sample were stained with hematoxylin (Merck 1.05174.0500) and eosin (Merck 109844) per the manufacturer's protocol.

For immunoblot analysis ${ }^{59}$, cells were lysed with RIPA lysis buffer containing $50 \mathrm{mM}$ Tris, $\mathrm{pH} 7.4,150 \mathrm{mM} \mathrm{NaCl}, 1 \% \mathrm{NP}-40$ (Sigma, 74385), 0.5\% sodium deoxycholate (Sigma, D6750), 0.1\% SDS (Sigma, L4509) with $200 \mu \mathrm{g} \mathrm{ml}^{-1}$ of phenylmethylsulfonyl fluoride (Sigma, P7626), phosphatase inhibitor cocktail 
(Sigma, P0044) and protease inhibitor cocktail (Millipore, 535140). The cell lysates were then resolved by $12 \%$ SDS-polyacrylamide gel electrophoresis, transferred onto Immobilon P membranes (Millipore, IPVH00010), and immunoblotted with anti-VCAM-1 Ab (Santa Cruz Biotechnology, Inc. sc-8304, dilution 1:1000), antiH3K27me3 Ab (EMD Millipore 07-449, dilution 1:1000), or anti-histone H3 Ab (Cell Signaling Technology 9715, dilution 1:1000) coupled with goat anti-rabbit IgG-HRP (Santa Cruz Biotechnology, Inc. sc-2004, dilution 1:2500), and anti-Phc2 $\mathrm{Ab}$ (mouse IgG, dilution 1:200) ${ }^{14}$ or anti- $\beta$-actin Ab (Sigma-Aldrich A5441, dilution 1:25,000) coupled with goat anti-mouse IgG-HRP (Santa Cruz Biotechnology, Inc. sc-2005, dilution 1:2500). Immunoreactive bands were visualized using an ECL solution (Thermo Fisher Scientific 34080). To quantify the relative protein expression, immunoreactive bands were normalized to levels of $\beta$ actin. The relative band intensity of protein expression was quantified using ImageJ software (National Institutes of Health). Uncropped blots can be found in the Source Data.

Cell cycle analysis and measurement of apoptosis. BM-resident LSK cells from 8 -week-old mice were isolated and incubated in $70 \%$ ethanol overnight at $4{ }^{\circ} \mathrm{C}$. After washing with PBS, cells were stained with propidium iodide (Sigma-Aldrich P4170) for cell cycle analysis. To measure apoptosis, LSK cells were stained with 7AAD (BD Biosciences 559925, dilution 1:20) and Annexin V-FITC (BD Biosciences 556547, dilution 1:20). After washing with PBS, stained cells were analyzed by flow cytometry.

CFU assay. Mononuclear cells isolated from the $\mathrm{BM}, \mathrm{PB}$, and spleen were plated onto Methocult GF M3434 (StemCell Technologies 03444) in $35 \mathrm{~mm}$ cell culture dishes. Cells were then incubated at $37^{\circ} \mathrm{C}$ and $5 \% \mathrm{CO}_{2}$. After 7 days of incubation, type and number of colonies were determined ${ }^{60}$.

In vivo HSPC mobilization assays. For G-CSF-induced mobilization assays ${ }^{15}$, recombinant human G-CSF (Amgen Filgrastim Neupogen) was diluted in PBS with $0.1 \%$ BSA (GenDEPOT A0100-050). G-CSF or vehicle was administered by daily subcutaneous injection to 8-week-old mice at a dose of $250 \mathrm{\mu g} \mathrm{kg}^{-1}$ day ${ }^{-1}$ for 5 days. For AMD3100-induced mobilization assays ${ }^{17}$, AMD3100 (Sigma-Aldrich A5602) was diluted in PBS and administered subcutaneously to 8-week-old mice at $5 \mathrm{mg} \mathrm{kg}^{-1}$. For G-CSF and AMD3100-induced mobilization assays ${ }^{61}$, G-CSF or vehicle was administered by daily subcutaneous injection to 8 -week-old mice at a dose of $250 \mu \mathrm{g} \mathrm{kg}^{-1}$ day $^{-1}$ for 5 days. One h before final G-CSF dose, AMD3100 administered subcutaneously to the same group of mice at $5 \mathrm{mg} \mathrm{kg}^{-1}$. Three $\mathrm{h}$ after the final G-CSF or AMD3100 dose, mice were sacrificed to assess the absolute numbers of nucleated cells in the PB and spleen. The absolute numbers of LSK cells in the $\mathrm{BM}, \mathrm{PB}$, and spleen were determined by flow cytometry and CFU assays. To block the VLA-4 and VCAM-1 interaction, anti-VCAM-1 (BD Biosciences 553330) $\mathrm{Ab}$ or the respective isotype control $\left(2 \mathrm{mg} \mathrm{kg}^{-1} \mathrm{day}^{-1}\right.$; BD Biosciences 553926) was administered intravenously every day.

For 5-FU-induced mobilization assays ${ }^{62}$, 5-FU (Sigma-Aldrich F6627) was administered intravenously to 8 -week-old mice at $200 \mathrm{mg} \mathrm{kg}^{-1}$. The absolute numbers of nucleated cells in the PB and spleen were counted on days $4,8,12$, and 16 after 5-FU treatment. On day 16 after 5-FU administration, mice were sacrificed, and the absolute numbers of $\mathrm{Lin}^{-} \mathrm{Sca}-{ }^{+}{ }^{+} \mathrm{c}-\mathrm{kit}^{+}$cells (LSK cells) in the $\mathrm{BM}, \mathrm{PB}$, and spleen were determined by flow cytometry and CFU assays. To block the VLA-4 and VCAM-1 interaction, mice were injected with anti-VCAM-1 Ab or the respective isotype control $\left(2 \mathrm{mg} \mathrm{kg}^{-1} \mathrm{day}^{-1}\right)$ from day 8 to day 15 after 5-FU administration.

For anti-VCAM-1 Ab-induced mobilization assays ${ }^{63}$, anti-VCAM-1 $\mathrm{Ab}$ or the respective isotype control administered by daily subcutaneous injection to 8-weekold mice at a dose of $2 \mathrm{mg} \mathrm{kg}^{-1} \mathrm{day}^{-1}$ for 3 days. Eight hours after the final $\mathrm{Ab}$ dose, mice were sacrificed, and the absolute numbers of nucleated cells in the PB and spleen were counted. The absolute numbers of LSK cells in the BM, PB, and spleen were determined by $\mathrm{CFU}$ assays described above.

LSK transplantation. LSK cells $\left(1 \times 10^{5}\right)$ isolated from 8-week-old donor mice were injected intravenously into lethally irradiated $(10 \mathrm{~Gy}) 8$-week-old recipient mice. Twelve weeks after the LSK transfer, recipient mice were sacrificed and analyzed for reconstitution of donor immune cells. Donor cells and recipient cells were discriminated by flow cytometry using anti-CD45.2 (104)-PerCP-Cy5.5 Ab (BD 552950, dilution 1:100) for congenic strain (CD45.1) discrimination. For serial competitive LSK repopulation assays ${ }^{58}$, LSK cells $\left(5 \times 10^{4}\right)$ isolated from WT or KO mice (CD45.2) were mixed with an equal number of LSK cells isolated from competitor mice (WT in CD45.1). The cell mixture was then intravenously injected into lethally irradiated (10 Gy) 8-week-old recipient mice (WT in CD45.1). Secondary transplantation was performed at 12 weeks after primary engraftment. LSK cells $\left(1 \times 10^{5}\right)$ harvested from primary transplants were intravenously injected into lethally irradiated (10 Gy) 8-week-old recipient mice (WT in CD45.1). The ratio of CD45.1 to CD45.2 positive cells in the BM, PB, thymus, and spleen of recipient mice was measured by flow cytometry.

In vivo homing assay. For in vivo homing assay ${ }^{64}$, recipient mice were lethally irradiated $(10 \mathrm{~Gy}) 24 \mathrm{~h}$ before LSK transplantation. Freshly isolated LSK cells from 8-week-old mice were labeled with $1 \mu \mathrm{M}$ CFSE (Thermo Fisher Scientific C34554) per the manufacturer's instructions. CFSE-labeled LSK cells $\left(2 \times 10^{5}\right)$ were then resuspended in PBS and intravenously injected into recipient mice. Sixteen hours after injection, mice were sacrificed, and the frequencies of $\mathrm{CFSE}^{+}$cells in the BM, $\mathrm{PB}$, and spleen were determined by flow cytometry. To determine the frequency of clonogenic progenitors, mononuclear cells from the BM, PB, and spleen were subjected to CFU assays as described above. To block the interaction between VLA4 and VCAM-1, CFSE-labeled LSK cells were incubated with an anti-VCAM-1 Ab $\left(2 \mathrm{mg} \mathrm{kg}^{-1}\right)$ or an isotype control, and the cells were intravenously injected into recipients $1 \mathrm{~h}$ before transplantation.

Trans-stromal migration and adhesion assay. For the trans-stromal migration assay ${ }^{65}$, LSK cells $\left(1 \times 10^{5}\right.$ cells chamber $\left.^{-1}\right)$ were loaded into the top chamber of a 24-well transwell plate containing a monolayer of BMSCs. Recombinant mouse CXCL12 (100 $\mathrm{ng} \mathrm{mL}^{-1}$; R\&D Systems P40224) was loaded into the bottom chamber. The plate was then incubated at $37^{\circ} \mathrm{C}$ in $5 \% \mathrm{CO}_{2}$ for $16 \mathrm{~h}$. After incubation, the number of migrating LSK cells in the bottom chamber was calculated. To perform the adhesion assay, CFSE-labeled LSK cells $\left(5 \times 10^{4}\right)$ were co-cultured with BMSCs $\left(2 \times 10^{5}\right)$ in 24 -well cell culture plates. Three $\mathrm{h}$ after co-culture, nonadherent LSK cells were removed via three gentle washes with PBS, and adherent LSK cells were harvested. The frequency of $\mathrm{CFSE}^{+}$LSK cells was determined by flow cytometry. To block the VLA-4 and VCAM-1 interaction, LSK cells were preincubated with anti-VLA-4 $\left(10 \mu \mathrm{g} \mathrm{mL}^{-1}\right.$; Merck CBL1304) Ab or an isotype control $\left(10 \mu \mathrm{g} \mathrm{ml}^{-1}\right.$; BD Biosciences 559478) for $30 \mathrm{~min}$, and BMSCs were preincubated with anti-VCAM-1 Ab $\left(10 \mu \mathrm{g} \mathrm{mL}^{-1}\right)$ or an isotype control for 30 min.

mRNA-seq data analysis. Total RNAs from WT and KO BM cells were extracted using TRIzol (Thermo Fisher Scientific 15596026) according to the manufacturer's protocol. Subsequently, $1 \mu \mathrm{g}$ of total RNA was used to construct cDNA libraries using TruSeq RNA library kit (Illumina, RS-122-2001) according to the manufacturer's protocol. The size distribution and quality of cDNA libraries were monitored by Agilent 2100 Bioanalyzer (Agilent Technologies) and quantitative PCR using Kapa Library Quantification Kit (Kapa Biosystems KK4824). After the quality check, cDNA libraries were sequenced using an Illumina

HiSeq4000 sequencer (Illumina).

After removing low quality and adapter sequences, the raw reads were aligned to the reference genome Mus musculus (mm10) (genome assembly information access, GCF_000001635.20) using HISAT v2.0.5 ${ }^{66}$. After aligning reads to the genome, StringTie v1.3.3b was used to assemble transcripts and estimate their abundance measured in fragments per kilobase of exon per million fragments mapped (FPKM) ${ }^{67,68}$. False discovery rate (FDR) was controlled by adjusting $p$-value cutoff of 0.05 using Benjamini-Hochberg algorithm ${ }^{69}$. Subsequently, hierarchical clustering analysis was performed to analyze differentially expressed gene (DEG) set using complete linkage and Euclidean distance as a measure of similarity. Gene set enrichment analysis including functional annotation and pathway analyses were performed based on Gene Ontology (www.geneontology.org/) and KEGG pathway (http://www.genome.jp/kegg/pathway.html). An overview of the gene expression data was deposited at NCBI's Gene Expression Omnibus (GEO). It is accessible through GEO series accession number of GSE128705.

Real-time quantitative RT-PCR analysis. Total RNA was purified from BMSCs using Trizol reagent (Thermo Fisher Scientific 15596026) according to the manufacturer's instructions; it was then reverse transcribed into cDNA using a RevertAid First Strand cDNA Synthesis Kit (Thermo Fisher Scientific K1621). After cDNA synthesis, quantitative PCR (qPCR) was conducted to measure mRNA levels of mouse Alcam, Angpt1, Cdh2, Ctsk, Cxcl12, Fn1, Kitl, Icam1, p16INK4a, $p 19^{A R F}$, Selp, Vcam1, and Gapdh using a 7500 Real-time PCR system (Thermo Fisher Scientific). Gapdh was used as an internal control to quantify the levels of other mRNA transcripts. The primer pairs used for qRT-PCR are listed in Supplementary Table 1.

Inhibition of Phc2 expression by shRNA. Lentiviral plasmids expressing shRNAs directed against several distinct regions of Phc2 (SHCLNG-NM_018774) and control shRNA plasmids (SHC001) were purchased from Sigma-Aldrich. OP9 cells (mouse BMSC line, ATCC CRL-2749) were transfected with each shRNAcontaining plasmid using Lipofectamine 2000 transfection reagent (Thermo Fisher Scientific 11668019) according to the manufacturer's instructions. After transfection, inhibition of Phc2 expression was determined by qRT-PCR and immunoblotting. Primer sequences for Phc2 for qRT-PCR are listed in Supplementary Table 1.

ChIP assay. The lysates of BM cells $\left(2 \times 10^{6}\right)$ from 8 -week-old mice were prepared for the ChIP assay ${ }^{70}$. BM cell lysates were sonicated and immunoprecipitated with the anti-Bmil (Santa Cruz Biotechnology, Inc. sc-10745, $2 \mu$ sample $^{-1}$ ), anti-Cbx7 (Santa Cruz Biotechnology, Inc. sc-70232, $2 \mu \mathrm{g} \mathrm{sample}^{-1}$ ), anti-H2AK119ub (Cell Signaling Technology 8240, $2 \mu \mathrm{g} \mathrm{sample}{ }^{-1}$ ), anti-H3K9ac (Merck 06-942, $2 \mu \mathrm{g}$ sample $^{-1}$ ), anti-H3K4me3 (Abcam ab12209, $2 \mu \mathrm{g} \mathrm{sample}^{-1}$ ), anti-H3K27me3 (Merck 07-449, $2 \mu \mathrm{g}$ sample ${ }^{-1}$ ), anti-Phc2 (Santa Cruz Biotechnology, Inc. sc- 
160664, $2 \mu \mathrm{g}$ sample ${ }^{-1}$ ), and anti-Ringlb (Cell Signaling Technology 5694, $2 \mu \mathrm{g}$ sample ${ }^{-1}$ ) Abs. Additionally, GSK126-treated OP9 cell lysates were sonicated and immunoprecipitated with the anti-Bmil, anti-Phc2, and anti-Ringlb Abs. After immunoprecipitation, immune complexes were collected with protein A agarose (Merck GE17-0963-03) and extracted with an extraction buffer (1\% SDS, $0.1 \mathrm{M}$ $\mathrm{NaHCO}_{2}$ ). DNA cross-links were reversed by heating to $65^{\circ} \mathrm{C}$ for $8 \mathrm{~h}$. DNA was extracted with phenol/chloroform and precipitated with ethanol. DNA isolated from an aliquot of total nuclear extract was used as the loading control for PCR (input control). qPCR was performed as described above. Data are presented after normalizing each immunoprecipitated DNA Ct value to $10 \%$ of the input DNA Ct value.

In vivo administration of GSK126. WT mice were intraperitoneally injected with 50 or $100 \mathrm{mg} \mathrm{kg}^{-1}$ of DMSO-dissolved GSK126. Mice were sacrificed at $16 \mathrm{~h}$ after GSK126 injection and the expression of VCAM-1 from BMSCs of sacrificed mice were analyzed by immunoblotting and flow cytometry. In addition, trans-stromal migration and adhesion assays using LSK cells and BMSCs of sacrificed mice were performed as described above.

Statistical analysis. Mean values among more than three experimental groups were compared by one-way ANOVA with Tukey HSD analysis and comparison between two groups was analyzed by two-tailed Student's $t$-test using SPSS statistics software, ver. 24.0 (SPSS Inc). All data are presented as the mean \pm standard error of mean (SEM). In each graph, significant differences as determined by $p$ values less than 0.05 and 0.01 are indicated by asterisks ( ${ }^{*}$ and ${ }^{* *}$, respectively).

Reporting summary. Further information on research design is available in the Nature Research Reporting Summary linked to this article.

\section{Data availability}

The raw mRNA-seq data were deposited into the Sequence Read Archive (SRA) of National Center for Biotechnology Information (NCBI) with the following accession numbers: GSM3683312, GSM3683313, GSM3683314, GSM3683315, GSM3683316, and GSM3683317. An overview of the gene expression data was deposited at NCBI's Gene Expression Omnibus (GEO). It is accessible through GEO series accession number of GSE128705. Source data underlying Figs. 1-8, Tables 1-2, Supplementary Figs. 1-7, and Supplementary Figs. 10-18 are provided as a Source Data file. All other data that support the findings of this study are available from the corresponding author upon reasonable request.

Received: 18 April 2018 Accepted: 12 July 2019

Published online: 02 August 2019

\section{References}

1. Wright, D. E., Wagers, A. J., Gulati, A. P., Johnson, F. L. \& Weissman, I. L. Physiological migration of hematopoietic stem and progenitor cells. Science 294, 1933-1936 (2001)

2. Laird, D. J., von Andrian, U. H. \& Wagers, A. J. Stem cell trafficking in tissue development, growth, and disease. Cell 132, 612-630 (2008).

3. Bhattacharya, D., Rossi, D. J., Bryder, D. \& Weissman, I. L. Purified hematopoietic stem cell engraftment of rare niches corrects severe lymphoid deficiencies without host conditioning. J. Exp. Med. 203, 73-85 (2006).

4. Morrison, S. J. \& Spradling, A. C. Stem cells and niches: mechanisms that promote stem cell maintenance throughout life. Cell 132, 598-611 (2008).

5. Mazo, I. B., Massberg, S. \& von Andrian, U. H. Hematopoietic stem and progenitor cell trafficking. Trends Immunol. 32, 493-503 (2011).

6. Wilson, A. \& Trumpp, A. Bone-marrow haematopoietic-stem-cell niches. Nat. Rev. Immunol. 6, 93-106 (2006).

7. Mercier, F. E., Ragu, C. \& Scadden, D. T. The bone marrow at the crossroads of blood and immunity. Nat. Rev. Immunol. 12, 49-60 (2012).

8. Piunti, A. \& Shilatifard, A. Epigenetic balance of gene expression by Polycomb and COMPASS families. Science 352, aad9780 (2016).

9. Cao, R. et al. Role of histone H3 lysine 27 methylation in Polycomb-group silencing. Science 298, 1039-1043 (2002).

10. Gao, Z. et al. PCGF homologs, CBX proteins, and RYBP define functionally distinct PRC1 family complexes. Mol. Cell. 45, 344-356 (2012).

11. Morey, L., Aloia, L., Cozzuto, L., Benitah, S. A. \& Di Croce, L. RYBP and Cbx7 define specific biological functions of polycomb complexes in mouse embryonic stem cells. Cell Rep. 3, 60-69 (2013).

12. Wang, H. et al. Role of histone H2A ubiquitination in Polycomb silencing. Nature 431, 873-878 (2004).
13. Tavares, L. et al. RYBP-PRC1 complexes mediate H2A ubiquitylation at polycomb target sites independently of PRC2 and H3K27me3. Cell 148 , 664-678 (2012)

14. Isono, K. et al. Mammalian polyhomeotic homologues Phc2 and Phcl act in synergy to mediate polycomb repression of Hox genes. Mol. Cell. Biol. 25, 6694-6706 (2005).

15. Petit, I. et al. G-CSF induces stem cell mobilization by decreasing bone marrow SDF-1 and up-regulating CXCR4. Nat. Immunol. 3, 687-694 (2002).

16. Bendall, L. J. \& Bradstock, K. F. G-CSF: from granulopoietic stimulant to bone marrow stem cell mobilizing agent. Cytokine Growth Factor Rev. 25, 355-367 (2014).

17. Broxmeyer, H. E. et al. Rapid mobilization of murine and human hematopoietic stem and progenitor cells with AMD3100, a CXCR4 antagonist. J. Exp. Med. 201, 1307-1318 (2005).

18. Devine, S. M. et al. Rapid mobilization of functional donor hematopoietic cells without G-CSF using AMD3100, an antagonist of the CXCR4/SDF-1 interaction. Blood 112, 990-998 (2008).

19. Heissig, B. et al. Recruitment of stem and progenitor cells from the bone marrow niche requires MMP-9 mediated release of kit-ligand. Cell 109, 625-637 (2002).

20. Aicher, A. et al. Essential role of endothelial nitric oxide synthase for mobilization of stem and progenitor cells. Nat. Med. 9, 1370-1376 (2003).

21. Calvi, L. et al. Osteoblastic cells regulate the haematopoietic stem cell niche. Nature 425, 841-846 (2003).

22. Kiel, M. J. et al. SLAM family receptors distinguish hematopoietic stem and progenitor cells and reveal endothelial niches for stem cells. Cell 121, 1109-1121 (2005)

23. Méndez-Ferrer, S. et al. Mesenchymal and haematopoietic stem cells form a unique bone marrow niche. Nature 466, 829-834 (2010).

24. Iademarco, M. F., Barks, J. L. \& Dean, D. C. Regulation of vascular cell adhesion molecule-1 expression by IL- 4 and TNF-alpha in cultured endothelial cells. J. Clin. Invest. 95, 264-271 (1995).

25. McCabe, M. T. et al. EZH2 inhibition as a therapeutic strategy for lymphoma with EZH2-activating mutations. Nature 492, 108-112 (2012).

26. Koni, P. A. et al. Conditional vascular cell adhesion molecule 1 deletion in mice: impaired lymphocyte migration to bone marrow. J. Exp. Med. 193, 741-754 (2001).

27. Ulyanova, T. et al. VCAM-1 expression in adult hematopoietic and nonhematopoietic cells is controlled by tissue-inductive signals and reflects their developmental origin. Blood 106, 86-94 (2005).

28. Scott, L. M., Priestley, G. V. \& Papayannopoulou, T. Deletion of a4 integrins from adult hematopoietic cells reveals roles in homeostasis, regeneration, and homing. Mol. Cell. Biol. 23, 9349-9360 (2003).

29. Priestley, G. V., Ulyanova, T. \& Papayannopoulou, T. Sustained alterations in biodistribution of stem/progenitor cells in Tie2Cre + a $4 \mathrm{f} / \mathrm{f}$ mice are hematopoietic cell autonomous. Blood 109, 109-111 (2007).

30. Papayannopoulou, T., Priestley, G. V., Nakamoto, B., Zafiropoulos, V. \& Scott, L. M. Molecular pathways in bone marrow homing: dominant role of $\alpha 4 \beta 1$ over $\beta 2$-integrins and selectins. Blood 98, 2403-2411 (2001).

31. Papayannopoulou, T. \& Nakamoto, B. Peripheralization of hemopoietic progenitors in primates treated with anti-VLA4 integrin. Proc. Natl Acad. Sci. USA 90, 9374-9378 (1993).

32. Kavanagh, D. P. et al. Haematopoietic stem cell recruitment to injured murine liver sinusoids depends on $\alpha 4 \beta 1$ integrin/VCAM-1 interactions. Gut 59, 79-87 (2010).

33. Osborn, L. et al. Direct expression cloning of vascular cell adhesion molecule 1, a cytokine-induced endothelial protein that binds to lymphocytes. Cell $\mathbf{5 9}$, 1203-1211 (1989).

34. Miyake, K. et al. A VCAM-like adhesion molecule on murine bone marrow stromal cells mediates binding of lymphocyte precursors in culture. J. Cell Biol. 114, 557-565 (1991).

35. Jacobsen, K., Kravitz, J., Kincade, P. \& Osmond, D. Adhesion receptors on bone marrow stromal cells: in vivo expression of vascular cell adhesion molecule- 1 by reticular cells and sinusoidal endothelium in normal and gamma-irradiated mice. Blood 87, 73-82 (1996).

36. Schweitzer, K. M. et al. Constitutive expression of E-selectin and vascular cell adhesion molecule-1 on endothelial cells of hematopoietic tissues. Am. J. Pathol. 148, 165-175 (1996).

37. Freedman, A. S. et al. Adhesion of human B cells to germinal centers in vitro involves VLA-4 and INCAM-110. Science 249, 1030-1033 (1990).

38. Schlesinger, M. \& Bendas, G. Vascular cell adhesion molecule-1 (VCAM-1) an increasing insight into its role in tumorigenicity and metastasis. Int. J. Cancer 136, 2504-2514 (2015).

39. Bradstock, K. F. \& Gottlieb, D. J. Interaction of acute leukemia cells with the bone marrow microenvironment: implications for control of minimal residual disease. Leuk. Lymphoma 18, 1-16 (1995). 
40. Winter, S. S. et al. Enhanced T-lineage acute lymphoblastic leukaemia cell survival on bone marrow stroma requires involvement of LFA-1 and ICAM-1. Br. J. Haematol. 115, 862-871 (2001).

41. Damiano, J. S., Cress, A. E., Hazlehurst, L. A., Shtil, A. A. \& Dalton, W. S. Cell adhesion mediated drug resistance (CAM-DR): role of integrins and resistance to apoptosis in human myeloma cell lines. Blood 93, 1658-1667 (1999).

42. Tabe, Y. et al. Activation of integrin-linked kinase is a critical prosurvival pathway induced in leukemic cells by bone marrow-derived stromal cells. Cancer Res. 67, 684-694 (2007).

43. Jacamo, R. et al. Reciprocal leukemia-stroma VCAM-1/VLA-4-dependent activation of NF- $\mathrm{KB}$ mediates chemoresistance. Blood 123, 2691-2702 (2014).

44. Cullen, S. M., Mayle, A., Rossi, L. \& Goodell, M. A. Hematopoietic stem cell development: an epigenetic journey. Curr. Top. Dev. Biol. 107, 39-75 (2014).

45. Hu, D. \& Shilatifard, A. Epigenetics of hematopoiesis and hematological malignancies. Genes Dev. 30, 2021-2041 (2016).

46. Takamatsu-Ichihara, E. \& Kitabayashi, I. The roles of Polycomb group proteins in hematopoietic stem cells and hematological malignancies. Int. J. Hematol. 103, 634-642 (2016).

47. Vidal, M. \& Starowicz, K. Polycomb complexes PRC1 and their function in hematopoiesis. Exp. Hematol. 48, 12-31 (2017).

48. Sato, T. et al. Mammalian Polycomb complexes are required for Peyer's patch development by regulating lymphoid cell proliferation. Gene 379, 166-174 (2006).

49. Ohta, H. et al. Polycomb group gene rae28 is required for sustaining activity of hematopoietic stem cells. J. Exp. Med. 195, 759-770 (2002).

50. Kim, J. Y. et al. Defective long-term repopulating ability in hematopoietic stem cells lacking the polycomb-group gene rae28. Eur. J. Haematol. 73, 75-84 (2004).

51. Hidalgo, I. et al. Ezh1 is required for hematopoietic stem cell maintenance and prevents senescence-like cell cycle arrest. Cell Stem Cell 11, 649-662 (2012).

52. Xie, H. et al. Polycomb repressive complex 2 regulates normal hematopoietic stem cell function in a developmental-stage-specific manner. Cell Stem Cell 14, 68-80 (2014)

53. van Den Boom, V. et al. Nonredundant and locus-specific gene repression functions of PRC1 paralog family members in human hematopoietic stem/ progeitor cells. Blood 121, 2452-2461 (2013).

54. Klauke, K. et al. Polycomb Cbx family members mediate the balance between haematopoietic stem cell self-renewal and differentiation. Nat. Cell Biol. 15, 353-362 (2013).

55. Gray, F. et al. BMI1 regulates PRC1 architecture and activity through homoand hetero-oligomerization. Nat. Commun. 7, 13343 (2016).

56. Peister, A. et al. Adult stem cells from bone marrow (MSCs) isolated from different strains of inbred mice vary in surface epitopes, rates of proliferation, and differentiation potential. Blood 103, 1662-1668 (2004).

57. Broske, A. M. et al. DNA methylation protects hematopoietic stem cell multipotency from myeloerythroid restriction. Nat. Genet. 41, 1207-1215 (2009).

58. Vukovic, M. et al. Adult hematopoietic stem cells lacking Hif-1 $\alpha$ self-renew normally. Blood 127, 2841-2846 (2016).

59. Han, J. et al. Autophagy induced by AXL receptor tyrosine kinase alleviates acute liver injury via inhibition of NLRP3 inflammasome activation in mice. Autophagy 12, 2326-2343 (2016).

60. Goldman, D. C. et al. BMP4 regulates the hematopoietic stem cell niche. Blood 114, 4393-4401 (2009).

61. Ramirez, P. et al. BIO5192, a small molecule inhibitor of VLA-4, mobilizes hematopoietic stem and progenitor cells. Blood 114, 1340-1343 (2009).

62. Chen., Z. et al. Wip1 deficiency impairs haematopoietic stem cell function via p53 and mTORC1 pathways. Nat. Commun. 6, 6808 (2015).

63. Papayannopoulou, T., Priestley, G. V. \& Nakamoto, B. Anti-VLA4/VCAM-1induced mobilization requires cooperative signaling through the kit/mkit ligand pathway. Blood 91, 2231-2239 (1998).

64. Foudi, A. et al. Reduced retention of radioprotective hematopoietic cells within the bone marrow microenvironment in $\mathrm{CXCR4^{-/- }}$ chimeric mice. Blood 107, 2243-2251 (2006).

65. Gonzalez-Nieto, D. et al. Connexin- 43 in the osteogenic BM niche regulates its cellular composition and the bidirectional traffic of hematopoietic stem cells and progenitors. Blood 119, 5144-5154 (2012).
66. Kim, D., Langmead, B. \& Salzberg, S. L. HISAT: a fast spliced aligner with low memory requirements. Nat. Methods 12, 357-360 (2015).

67. Pertea, M. et al. StringTie enables improved reconstruction of a transcriptome from RNA-seq reads. Nat. Biotechnol. 33, 290-295 (2015).

68. Pertea, M., Kim, D., Pertea, G. M., Leek, J. T. \& Salzberg, S. L. Transcript-level expression analysis of RNA-seq experiments with HISAT, StringTie and Ballgown. Nat. Protoc. 11, 1650-1667 (2016).

69. Benjamini, Y. \& Hochberg, Y. Controlling the false discovery rate: a practical and powerful approach to multiple testing. J. R. Soc. Ser. B Stat. Methodol. 57, 289-300 (1995)

70. Cho, K. W., Bae, J., Lee, S. J. \& Chun, T. Expression pattern and functional role of Phc2 during activation of helper T cells after antigenic stimulation. In Vitro Cell Dev. Biol. Anim 49, 360-370 (2013).

\section{Acknowledgements}

We thank Amgen Inc. (Thousand Oaks, CA, USA) for providing G-CSF. We also thank Eun-Kyeong Jo (Chungnam National University School of Medicine), Kyung-Mi Lee (Korea University College of Medicine), Seungkwon You (Korea University School of Life Sciences and Biotechnology), and Seok-Ho Hong (Kangwon National University College of Medicine) for their critical reading of our manuscript or helpful suggestions. This research was supported by the Bio \& Medical Technology Development Program of the National Research Foundation (NRF) funded by the Ministry of Science \& ICT (2017M3A9C8060392), and an intramural research program funded by Korea University grant (2016).

\section{Author contributions}

T.C. conceived, designed, and supervised this study. J.B., S.-P.C., K.I., J.Y.L., S.-W.P., C.-Y.C., J.H., S.-H.K., H.-H.L., K.P. and S.J.L. performed experiments. J.B., S.-P.C. C.-Y.C., C.-G.P. and T.C. analyzed and interpreted data. J.B., S.-P.C., H.Y.J., C.-G.P., H.K., Y.S.L. and T.C. discussed, wrote and edited the manuscript. All authors reviewed and approved the manuscript.

\section{Additional information}

Supplementary Information accompanies this paper at https://doi.org/10.1038/s41467019-11386-4.

Competing interests: The authors declare no competing interests.

Reprints and permission information is available online at http://npg.nature.com/ reprintsandpermissions/

Peer review information: Nature Communications thanks Hal Broxmeyer and other anonymous reviewer(s) for their contribution to the peer review of this work. Peer reviewer reports are available.

Publisher's note: Springer Nature remains neutral with regard to jurisdictional claims in published maps and institutional affiliations.

Open Access This article is licensed under a Creative Commons By Attribution 4.0 International License, which permits use, sharing,
adaptation, distribution and reproduction in any medium or format, as long as you give appropriate credit to the original author(s) and the source, provide a link to the Creative Commons license, and indicate if changes were made. The images or other third party material in this article are included in the article's Creative Commons license, unless indicated otherwise in a credit line to the material. If material is not included in the article's Creative Commons license and your intended use is not permitted by statutory regulation or exceeds the permitted use, you will need to obtain permission directly from the copyright holder. To view a copy of this license, visit http://creativecommons.org/ licenses/by/4.0/.

(C) The Author(s) 2019 